\title{
Gründe und Ausformungen des Euroskeptizismus bei der Republikanischen Volkspartei (CHP) und bei der Nationalistischen Bewegungspartei (MHP)
}

Causes and manifestations of Euroskepticism in the Republican People's Party (CHP) and the Nationalist Movement Party (MHP)

\section{Can Büyükbay}

\section{OpenEdition}

\section{Journals}

Édition électronique

URL : http://journals.openedition.org/ejts/4370

DOI : $10.4000 /$ ejts.4370

ISSN : $1773-0546$

Éditeur

EJTS

Référence électronique

Can Büyükbay, «Gründe und Ausformungen des Euroskeptizismus bei der Republikanischen

Volkspartei (CHP) und bei der Nationalistischen Bewegungspartei (MHP) », European Journal of Turkish Studies [En ligne], Liste complète, mis en ligne le 22 juin 2011, consulté le 19 février 2020. URL : http:// journals.openedition.org/ejts/4370 ; DOI : 10.4000/ejts.4370

Ce document a été généré automatiquement le 19 février 2020.

(c) Some rights reserved / Creative Commons license 


\section{Gründe und Ausformungen des Euroskeptizismus bei der} Republikanischen Volkspartei (CHP) und bei der Nationalistischen Bewegungspartei (MHP)

Causes and manifestations of Euroskepticism in the Republican People's Party (CHP) and the Nationalist Movement Party (MHP)

\section{Can Büyükbay}

\section{EINLEITUNG}

1 Die Anerkennung der Türkei als Beitrittskandidat auf dem Helsinki-Gipfel im Jahr 1999 hat im türkischen politischen System sowohl die Reformprozesse im Rahmen der Kopenhagener Kriterien beschleunigt, als auch euroskeptische Haltungen bei den politischen Akteuren hervorgerufen. Obwohl die türkische Elite behauptet, heute grundsätzlich eine pro-europäische Haltung einzunehmen, die nach dem Verständnis des Staatsgründers Mustafa Kemal Atatürk als Verwestlichung verstanden werden könnte, verschleiert diese grundlegende pro-europäische Haltung nicht die Tatsache, dass die türkische Elite aus verschiedenen Gründen eine Skepsis gegenüber der Europäischen Union entwickelt hat. Infolgedessen kann festgestellt werden, dass der Europäisierungsprozess der Türkei nach der „Post-Helsinki-Ära“ ${ }^{\text {“1 }}$ im türkischen Parteiensystem nicht nur Enthusiasmus, sondern auch viel Skepsis auslöste. So trug der Helsinki-Gipfel zu einer Neuordnung des türkischen Parteiensystems bei. Das türkische Parteiensystem wird durch eine neue Konfliktlinie geteilt: Neben dem klassischen Rechts-Links-Schema unterscheiden sich die Parteien jetzt noch zusätzlich durch proeuropäische beziehungsweise euroskeptische Positionen (Öniş 2003: 16). Die CHP (Republikanische Volkspartei) und die MHP (Nationalistische Bewegungspartei) sind 
neben der Regierungspartei AKP (Partei für Gerechtigkeit und Entwicklung) und der pro-kurdischen Partei DTP ${ }^{2}$ (Partei der demokratischen Gesellschaft) im Hinblick auf ihre Ideologien und Auswirkungen die wichtigsten Akteure im Parteiensystem der Türkei und können somit dem Beitrittsprozess einen Impuls geben oder ihn verhindern. Die Positionen der AKP und der DTP waren eher pro-europäisch. Der Reformprozess, der von der Regierungspartei AKP seit 2002 lanciert wurde, wurde in der Öffentlichkeit als „stille Revolution“ bezeichnet. Paradoxerweise war es die AKP, die einen islamischen Hintergrund hat und deren Vorgänger starke Europagegner waren, die die radikalen Demokratisierungsreformen von 2002 bis 2005 durchgesetzt hatte. Die AKP überraschte viele Beobachter und begann mit Änderungen der Rechtsvorschriften, die einen demokratischen, liberalen und progressiven Charakter hatten. Der Reformeifer der AKP nahm jedoch ab 2005 deutlich ab. Die Kritiker der AKP argumentierten, dass die Regierungspartei ihre Regierungsmacht gesichert habe und sie die EU für eine Erweiterung ihres Handlungsspielraums in religiösen Fragen nicht mehr brauche. Die DTP war eine pro-kurdische Partei und wurde im Jahr 2005 gegründet. Sie unterstützte den EU-Integrationsprozess insbesondere wegen der in der EU betonten Wichtigkeit der politischen und sozialen Rechte der kurdischen Minderheit. Aus diesem Grund wurde die EU-Integration generell als eine umfassende Demokratisierung der Türkei wahrgenommen. Diese beiden Parteien können im erwähnten Zeitraum dem pro-EU Lager zugeordnet werden.

2 Der vorliegende Artikel wird auf die linke sozialdemokratische Republikanische Volkspartei (CHP) und die rechte Nationalistische Bewegungspartei (MHP) begrenzt, die dem europäischen Integrationsprojekt entweder grundsätzlich oder in seiner gegenwärtigen Form skeptisch gegenüberstehen und sich infolgedessen mit ideologischen, institutionellen und strategischen Herausforderungen konfrontiert sehen. Es muss auch geklärt werden, warum eine Fokussierung auf die politischen Parteien in der Türkei notwendig ist: Die Aussicht auf eine EU-Mitgliedschaft kann ein starker Antrieb für Demokratisierung und wirtschaftlichen Wandel in den Beitrittsländern sein. Obwohl die EU ein wichtiger externer Faktor für den innenpolitischen Wandel ist, müssen zuerst die innenpolitischen Akteure den Anstoß für eben diesen Wandel geben. Die Analyse des innenpolitischen Prozesses in der Türkei ist außerdem wichtig, weil sich die institutionellen, politischen und normativen Rahmenbedingungen, die den Euroskeptizismus bei den politischen Parteien in der Türkei beeinflussen, in erheblichem Maße von denen der europäischen politischen Parteien unterscheiden. Obwohl in der politikwissenschaftlichen Forschung der letzten Jahre den Positionen, die die Parteien gegenüber der europäischen Integration und der Europäischen Union einnehmen, immer mehr Aufmerksamkeit geschenkt wurde, bleibt der türkische Fall insgesamt weitgehend unbeachtet. Die Positionen der türkischen politischen Parteien gegenüber der europäischen Integration und der Europäischen Union müssen detailliert betrachtet werden, da die Parteien aufgrund ihres direkten negativen bzw. positiven Einflusses die wichtigsten Akteure des Beitrittsprozesses der Türkei sind. In diesem Artikel wird deswegen versucht, den bis jetzt wenig untersuchten parteibasierten Euroskeptizismus in der Türkei bei der MHP und der CHP im Zeitabschnitt 2004-2007 zu analysieren und die Gründe für eine negative EUEinstellung bei diesen Parteien festzustellen. Die Fragestellung dieser Arbeit lautet demnach:

„Welche Ausformungen des Euroskeptizismus haben die CHP und die MHP und welche Gemeinsamkeiten und Unterschiede sind bei diesen Parteien hinsichtlich 
des Euroskeptizismus festzustellen? Was sind die Ursachen der Unterschiede und Gemeinsamkeiten bei den Haltungen der beiden Parteien?“

Der Artikel ist wie folgt gegliedert: Nach der Einleitung werden im theoretischen Teil der parteibasierte Euroskeptizismus und dessen Gründe vorgestellt. Dabei wird auf die von Kopecky und Mudde (2002) entwickelte Typologie des parteibasierten Euroskeptizismus zurückgegriffen. Im dritten Kapitel werden die qualitative Analyse nach Mayring (2008) und die Differenz- und Konkordanzmethode erläutert, die dazu dienen, eine systematische Analyse der Dokumente zu ermöglichen und einen wissenschaftlichen Vergleich zwischen der CHP und der MHP zu ziehen. Im vierten Kapitel wird in Anlehnung an die vierfache Typologie von Kopecky und Mudde ein Kategoriensystem erstellt und die Analyse der Parteiprogramme und der verschiedenen Dokumente durchgeführt, die zur Feststellung der Ausformungen des Euroskeptizismus und dem Vergleich beider Parteien dienen sollen. Zum Schluss folgt im fünften Kapitel der kontrollierte Vergleich des Euroskeptizismus beider Parteien anhand der Differenzmethode von John Stuart Mill.

\section{THEORIE}

4 In diesem Kapitel wird die vierfache Typologie von Kopecky und Mudde (2002) vorgestellt. Anschließend werden verschiedene Erklärungsansätze in der Forschung für die Gründe des Euroskeptizismus diskutiert.

\section{Euroskeptizismus nach Kopecky und Mudde}

Mit einer Rückkehr zum ursprünglichen Konzept von Taggart (1998), bei dem der Schwerpunkt eher auf der Haltung gegenüber der europäischen Integration liegt, entwickeln Kopecky und Mudde eine zweistufige Differenzierung. Sie berufen sich auf David Eastons bahnbrechende Unterscheidung der verschiedenen Formen bezüglich der Unterstützung des politischen Regimes (Easton 1965: 124ff. zit. nach Kopecky \& Mudde 2002: 300) und betrachten dabei separat die diffuse und spezifische Unterstützung der europäischen Integration. Während sich die diffuse Unterstützung auf die grundlegenden Ideen der europäischen Integration bezieht, ist mit der spezifischen Unterstützung die Praktik der europäischen Integration gemeint.

Tabelle 2: Das vierfache Modell des parteibasierten Euroskeptizismus

Support for idea of European Integration

\begin{tabular}{|l|l|l|l||}
\hline \multicolumn{1}{|l|}{} & \multicolumn{1}{|c|}{} & \multicolumn{1}{|c|}{ Europhile } & \multicolumn{1}{|c|}{ Europhobe } \\
\hline \hline \multirow{2}{*}{ Support for EU } & EU-Optimist & EUROENTHUSIATS & EUROPRAGMATISTS \\
\cline { 2 - 4 } & EU-Pessimist & EUROSCEPTICS & EUROREJECTS \\
\hline
\end{tabular}

(Kopecky \& Mudde 2002) 
"The breakdown of the concept of Euroscepticism into two analytical dimensions contributes to the clarification of the fundamental difference between parties that are critical only of the $\mathrm{EU}$, and those that are also negative about the ideas underlying the general process of European integration." (Kopecky \& Mudde 2002: 318)

\section{Die Frage der Kausalität: Gründe des Euroskeptizismus}

Es ist wichtig zu verstehen, warum die Parteien eine kritische oder ablehnende Haltung gegenüber der EU und der europäischen Integration haben. In der Literatur werden allgemein ideologisch-programmatische oder strategisch-taktische Faktoren herausgestellt. Viele Forscher (Taggart 1998; Taggart \& Szczerbiak 2001; Sitter 2001) argumentieren, dass Euroskeptizismus vornehmlich mit strategischen Faktoren, die von der Position der Partei im Parteiensystem abhängig sind, erklärt werden kann (zum Beispiel ob sich die Partei im Zentrum oder in der Peripherie des politischen Spektrums befindet oder ob sie der Regierung oder der Opposition angehört). Diese einflussreiche Gruppe von Forschern sieht den parteibasierten Euroskeptizismus als eine Frage der strategischen Positionierung und stellt ihn in Zusammenhang mit der sogenannten „Politik der Opposition“. Gemäß dieser Ansicht werden politische Parteien als "organizations that seek to propel candidates to elected office in pursuit of policy goals” (Sitter 2002: 5) betrachtet. Die Betonung liegt hier auf dem „quest for office", das das wichtigste Ziel einer politischen Partei ist.

7 Auf der anderen Seite gibt es Forscher, die die Parteienpositionen eher mit den ideologischen-programmatischen Faktoren erklären. Marks et al. (2002) gehen beispielsweise davon aus, dass die wichtigste Ursache für eine euroskeptische Haltung bei Parteien in historischen Spaltungen zu finden sind, welche zu den ideologischen Partei-Familien nach Lipset und Rokkan geführt haben: liberal, sozialdemokratisch, konservativ oder christdemokratisch (Taggart \& Szczerbiak 2003: 12). Kopecky und Mudde (2002: 320) betonen auch die Auswirkung von Ideologien auf die Einstellung von Parteien zur EU-Frage. Sie kommen zu dem Ergebnis, dass die Ideologie einer Partei die Haltung gegenüber der europäischen Integration grundsätzlich determiniert, während die Strategie die Unterstützung der gegenwärtigen Programme der EU bestimmt.

\section{METHODISCHES VORGEHEN}

$8 \quad$ Für die Zwecke dieses Artikels werden vor allem Analysen des politischen Diskurses in Betracht gezogen. Erstens: Eine Analyse der Parteiprogramme und der öffentlichen Auftritte der Parteifunktionäre soll einen Rahmen für das Verständnis der Art des Euroskeptizismus bei den entsprechenden politischen Parteien schaffen. Für die Analyse werden die Sitzungsprotokolle des türkischen Parlaments und die Veröffentlichungen der entsprechenden Parteien bezüglich ihrer Haltung zum Thema herangezogen. Zweitens: Die Artikel der Politologen in der Türkei und der Türkeispezialisten in Europa werden als wertvolle Quellen für die Analyse verwendet, um das Thema tiefgreifend und umfassend betrachten zu können. Die Methoden, die in der Arbeit verwendet werden, sind im Folgenden dargestellt:

Die qualitative Inhaltsanalyse nach Mayring (2008) ermöglicht eine systematische, theorie- und regelgeleitete Analyse der Parlamentsprotokolle und der Veröffentlichungen der CHP und MHP. Die systematische Auswertung der Texte 
erfordert klare Interpretationsregeln, um die intersubjektive Überprüfbarkeit zu gewähren.

Um einen kontrollierten Vergleich zwischen dem Euroskeptizismus bei der MHP und bei der CHP zu ziehen, werden die Konkordanzmethode und die Differenzmethode verwendet, die John Stuart Mill 1874 in seinem Werk „A System of Logic“ vorstellt. Auf der Basis einer deterministischen Kausalität wird bei diesen Methoden angenommen, dass jedes Ereignis eine Ursache hat. John Stuart Mill beschreibt (1874: 280) die Konkordanzmethode (Method of Agreement) folgendermaßen:

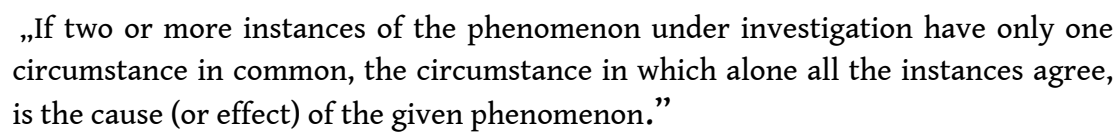

Die Konkordanzmethode, mit der notwendige Bedingungen bestimmt werden können, vergleicht Fälle, die mit dem Ergebnis, das man erklären will, übereinstimmen. Man versucht einen Kausalfaktor zu identifizieren, indem man nach Erklärungsfaktoren (unabhängige Variable) sucht, die in gleicher Weise wie das Ergebnis (abhängige Variable) auftreten. Sie bedient sich eines Ausschlussverfahrens, bis keine Option oder Ursache mehr übrig bleibt (Blatter et al. 2007: 142). Eine einzige Abweichung vom erwarteten Muster reicht aus, um eine Bedingung auszuschließen.

Die Differenzmethode vergleicht Fälle, die mit dem Ergebnis, das man erklären will, nicht übereinstimmen. Um einen Kausalfaktor zu identifizieren, sucht man nach Erklärungsfaktoren, die parallel zum Ergebnis variieren. Mit der Differenzmethode können hinreichende Bedingungen bestimmt werden. Mill beschreibt die Differenzmethode wie folgt:

„If an instance in which the phenomenon under investigation occurs, and an instance in which it does not occur, have every circumstance in common save one, that one occurring only in the former: the circumstance in which alone the two instances differ, is the effect, or the cause, or an indispensable part of the cause, of the phenomenon." (Mill 1874: 280)

\section{ANALYSE DES EUROSKEPTIZISMUS BEI DER MHP UND DER CHP (2004-2007)}

13 Bevor eine detaillierte Analyse erfolgen kann, müssen die Vorstellungen der MHP und CHP bezüglich der europäischen Integration dargestellt werden. Wie im theoretischen Teil erklärt wurde, kann dadurch bestimmt werden, ob die Parteien europhil oder europhob sind. Europhile glauben an die grundlegenden Ideen der europäischen Integration, die in zwei Bereiche unterteilt werden können: In den der institutionalisierten Kooperation auf der Basis der verkoppelten Souveränität als politisches Element einerseits und in den der integrierten Märkte als ökonomisches Element andererseits. Europhobe opponieren gegen die gesamte Idee der europäischen Integration. Nach Kopecky und Mudde (2002: 301) reicht es aus, wenn eine Partei nur eine der europäischen Integration zugrunde liegenden Grundideen nicht unterstützt, um sie als europhob zu bezeichnen. In der vorliegenden Arbeit wird als Unterscheidungskriterium eher die ideologische Dimension betrachtet, denn in Anlehnung an Eder (2004: 49) kann davon ausgegangen werden, dass in der Beziehung zwischen der Türkei und der EU, insbesondere im Zusammenhang mit den Kopenhagener Kriterien, die politische Dimension (Stabilität der Institutionen, die die 
Demokratie garantieren, Menschenrechte, Rechtsstaat, Schutz und Gewährung der Minderheitenrechte) im Gegensatz zur ökonomischen Dimension mehr Aufmerksamkeit auf sich gezogen hat. Dies benötigt eine Analyse der Themenbereiche, denen im türkischen Kontext hohe Bedeutung beigemessen wird.

An dieser Stelle sind einige wichtige Punkte zu erwähnen: Erstens betonen Kopecky und Mudde (2002: 302), dass eine Partei mit kritischer Haltung gegenüber einer spezifischen EU-Politik durchaus EU-optimistisch sein kann. Sobald eine Partei insgesamt die gegenwärtige EU akzeptiert, gehört sie zu dieser Kategorie. Zweitens ist es offensichtlich, dass die EU-Pessimisten die aktuelle Form der EU nicht unterstützen und ihrer Entwicklungsrichtung gegenüber skeptisch stehen. Dennoch bedeutet es nicht zwangsläufig, dass die EU-Pessimisten die EU-Mitgliedschaft ablehnen. Die aktuelle EU wird von manchen EU-Pessimisten als eine Deviation vom Gründungsgedanken der europäischen Integration bewertet. Weil sie aber diesen Gedanken unterstützen, hoffen sie auf eine Rückwandlung der EU zu dieser Idee.

\section{Die Durchführung der qualitativen Inhaltsanalyse}

Da der Fortschrittsbericht vom 6.0ktober 2004 und der Verhandlungsrahmen vom 3.Oktober 2005 bei der Europäischen Kommission in den beiden politischen Parteien viel Kritik hervorgerufen haben und ihren Euroskeptizismus bis heute prägen, ist der Untersuchungszeitraum 2004-2007 von besonderer Bedeutung. Der Euroskeptizismus nahm nach diesem Zeitpunkt bei beiden Parteien stabile Formen an. Nach einer ersten Analyse der Parteidokumente wird deutlich, dass die in diesem Zeitraum vorgebrachten Kritikpunkte gegen die EU als beispielhaft für beide Parteien angesehen werden können. Dem Fortschrittsbericht der EU-Kommission im Jahr 2006 schloss sich eine Debatte über den Artikel 301 an, die bis heute noch andauert. Eine Analyse der Parteiprogramme soll einen Rahmen für das Verständnis der Art des Euroskeptizismus bei den entsprechenden politischen Parteien schaffen. Ferner werden für die Analyse die Sitzungsprotokolle des türkischen Parlaments, die Veröffentlichungen der CHP und MHP bezüglich ihrer Haltung zum Thema und die wichtigsten repräsentativen Pressemitteilungen der Parteispitzen herangezogen. Das Material wurde mit spezieller Berücksichtigung des Untersuchungszeitraums 2004-2007 ausgewählt. Es wird darauf geachtet, genau spezifizierte Dokumente $z u$ verwenden, die die europapolitischen Positionen der CHP und der MHP reflektieren und die als repräsentativ für die Kritikpunkte der Parteien gegenüber der EU und der europäischen Integration in verschiedenen Themen gelten können. Die Kritikpunkte der Parteien bezüglich der Fortschrittsberichte der Kommission von 2004 und 2006 und dem Verhandlungsrahmen von 2005 bilden in dieser Periode den zentralen Rahmen für die Analyse des Euroskeptizismus. Da die MHP in dieser Periode nicht im Parlament vertreten war, wird in der Analyse hauptsächlich der Fokus werden auf das Parteiprogramm und die Veröffentlichungen der MHP bezüglich des EUBeitrittsprozesses gelegt. Das Buch „Ja zur Vollmitgliedschaft, nein zur privilegierten Partnerschaft" aus dem CHP-Zentrum in Ankara stellt die Einstellung der CHP zum EUBeitritt der Türkei ab dem Jahr 2002 dar. Es beinhaltet alle Presseerklärungen und Interviews der CHP-Abgeordneten mit Medienvertretern über die Europa-Frage und alle Gespräche des Vorsitzenden und der Abgeordneten der CHP, die in der eigenen Partei geführt wurden. Die Auswahl verschiedener Arten von Dokumenten dient auch 
dazu, die in den Europadiskursen auftretenden Konflikte innerhalb der jeweiligen Partei herauszufinden.

Den theoretischen Hintergrund des Kategoriensystems bildet die von Kopecky und Mudde vorgeschlagene Typologie des Euroskeptizismus. Um die Analyse in einzelne Interpretationsschritte zu zerlegen, wird ein Ablaufmodell eingesetzt (Mayring 2008: 53). Als Kodiereinheit dienen im weiteren Sinne EU-bezogene Aussagen der Produzenten der Texte. Als Auswertungseinheit gilt bei der Kodierung die Stellungnahme des jeweiligen Redners. Das Kategoriensystem legt fest, welche Aspekte aus dem Material herausgefiltert werden sollen. In dieser Arbeit werden die sortierten Themenbereiche nach der Form des Euroskeptizismus untersucht und in das Kategoriensystem eingeordnet. Stellungnahmen gegenüber der EU und der europäischen Integration wurden herausgefiltert. Im Folgenden wird der Kodierleitfaden vorgestellt:

Tabelle 2: Der Kodierleitfaden ${ }^{2}$ (Quelle: Kopecky und Mudde 2002 und eigene Überlegungen)

\begin{tabular}{|l|l|l|l|l|}
\hline Variable & Kategorie & Definition & Ankerbeispiel & Kodierregel \\
\hline
\end{tabular}




\begin{tabular}{|c|c|c|c|c|}
\hline \multirow[t]{4}{*}{ Euroskeptizismus } & $\begin{array}{l}\text { EU- } \\
\text { Enthusiasten } \\
\text { (europhile } \\
\text { Optimisten) }\end{array}$ & $\begin{array}{l}\text { Parteien, die die } \\
\text { europäische } \\
\text { Integration und } \\
\text { deren konkrete } \\
\text { Praxis } \\
\text { unterstützen. }\end{array}$ & $\begin{array}{l}\text { „Die CHP hat von je her die } \\
\text { EU-Mitgliedschaft der } \\
\text { Türkei unterstützt“ } \\
\text { (CHP 2008: 1) }\end{array}$ & $\begin{array}{l}\text { Im Parteiprogramm „ja“ } \\
\text { zur } \quad \text { Europäischen } \\
\text { Integration und den EU- } \\
\text { Anforderungen. } \\
\text { Kein Widerspruch } \\
\text { zwischen den Äußerungen } \\
\text { der Parteifunktionäre und } \\
\text { dem Parteiprogramm. } \\
\text {-Es können einige Aspekte } \\
\text { der EU. kritisiert werden, } \\
\text { wenn die generelle Form } \\
\text { der gegenwärtigen EU } \\
\text { akzeptiert wird. }\end{array}$ \\
\hline & $\begin{array}{l}\text { EU-Skeptiker } \\
\text { (europhile } \\
\text { Pessimisten) }\end{array}$ & $\begin{array}{l}\text { Parteien, die die } \\
\text { europäische } \\
\text { Integration } \\
\text { unterstützen, } \\
\text { aber deren } \\
\text { konkrete Praxis } \\
\text { kritisieren. }\end{array}$ & $\begin{array}{l}\text { „Im Falle [...], dass der } \\
\text { Beschluss } \\
\text { privilegierten } \\
\text { Mitgliedschaft der Türkei } \\
\text { zur offiziellen Position der } \\
\text { EU wird, so werden unsere } \\
\text { vorhandenen Zusagen, } \\
\text { angeführt von der } \\
\text { Zollunion, revidiert und } \\
\text { die gemäß den Vorteilen } \\
\text { unseres Landes } \\
\text { erforderlichen } \\
\text { Maßnahmen getroffen } \\
\text { werden“ } \\
\text { (CHP 2008: 3) }\end{array}$ & $\begin{array}{l}\text { Im Parteiprogramm ,ja“ } \\
\text { zur Europäischen } \\
\text { Integration, Kritik der EU- } \\
\text { Anforderungen Kontext. } \\
\text { bestimmten Themen im } \\
\text { türkischen den } \\
\text { Bewertung der EU als eine } \\
\text { Deviation von } \\
\text { grundlegenden Ideen der } \\
\text { europäischen Integration. } \\
\text { Kein } \\
\text { zwischen den Äußerungen } \\
\text { der Parteifunktionäre und } \\
\text { dem Parteiprogramm. } \\
\text { Die Äußerungen und das } \\
\text { Parteiprogramm sind nicht } \\
\text { gegen die grundsätzlichen } \\
\text { Ideen der europäischen } \\
\text { Integration. }\end{array}$ \\
\hline & $\begin{array}{l}\text { EU- } \\
\text { Pragmatiker } \\
\text { (europhobe } \\
\text { Optimisten) }\end{array}$ & $\begin{array}{l}\text { Parteien die } \\
\text { gegen die } \\
\text { europäische } \\
\text { Integration sind, } \\
\text { aber deren } \\
\text { Praxis } \\
\text { unterstützen. }\end{array}$ & - & $\begin{array}{l}\text { Im Parteiprogramm keine } \\
\text { Unterstützung für die } \\
\text { grundlegende Ideen der } \\
\text { europäischen Integration. } \\
\text { In den Äußerungen der } \\
\text { Parteifunktionären „ja“ } \\
\text { zur EU wegen } \\
\text { utilitaristischen } \\
\text { Kalkulationen. }\end{array}$ \\
\hline & $\begin{array}{l}\text { EU-Gegner } \\
\text { (europhobe } \\
\text { Pessimisten) }\end{array}$ & $\begin{array}{l}\text { Parteien, die die } \\
\text { europäische } \\
\text { Integration und } \\
\text { deren konkrete } \\
\text { Praxis ablehnen. }\end{array}$ & 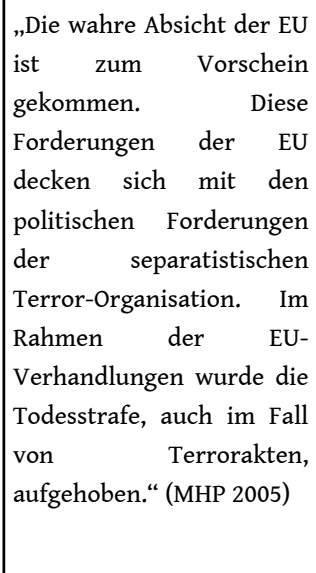 & $\begin{array}{l}\text { Im Parteiprogramm „nein“ } \\
\text { zur } \\
\text { Integration und der EU. } \\
\text { - Im } \\
\text { zur Parteiprogramm ,ja“ } \\
\text { Integration. } \\
\text { Äußerungen }\end{array}$ \\
\hline
\end{tabular}




\section{Analyse des Parteiprogramms der MHP}

Die Nationalistische Bewegungspartei (MHP) sieht die Türkei im Parteiprogramm als einen Verbündeten des Westens. Sie akzeptiert prinzipiell die Vollmitgliedschaft in der Europäischen Union, die sie als eine Staatspolitik der Türkischen Republik bewertet, und ist überzeugt davon, dass gegenseitige Zusammenarbeit und Verständnis die Beziehung beherrschen sollte. Die Beziehung zur Europäischen Union werden nicht nur als eine Beteiligung der Türkei an einer regionalen Formation bezeichnet, sondern definiert auch strategischen Präferenzen, die neue Entwicklungen in sozialen, wirtschaftlichen und internationalen Bereichen mit sich bringen werden. Die MHP bewertet die Beziehung zur EU in der Geschichte als einseitig und betont die Berücksichtigung gegenseitiger Interessen. Da es nicht möglich sei, die Beziehung zur Europäischen Union, wie in der Vergangenheit, durch einseitige Erwartungen und Kompromisse fortzuführen, solle eine Außenpolitik verfolgt werden, die sich gegen die Auferlegung von Pflichten der Türkei und für eine Berücksichtigung gegenseitiger Interessen ausspricht. Die MHP behauptet, dass sie keine Vorurteile gegen die europäische Integration hat. Die mögliche EU-Mitgliedschaft der Türkei wird im Parteiprogramm anerkannt, aber diese Integration möge in einer gerechten und ehrenhaften Art und Weise stattfinden. Es ist allerdings ersichtlich, dass es Vorbehalte hinsichtlich der nationalen Interessen und Sensibilitäten der Türkei gibt. Dies wird in der folgenden Textpassage deutlich:

„Die für das türkische Volk unverzichtbaren Faktoren sind Respekt und Gebundenheit an unsere National-Kultur, die Einheit unseres Landes und unsere einheitliche Staatsstruktur. Der Respekt, den die Länder der Europäischen Union gegenüber unseren nationalen Sensibilitäten zeigen werden, wird die Zukunft unserer Beziehungen zu diesen Ländern bestimmen." 4 (MHP 2008)

19 Als unverzichtbare „nationale Sensibilitäten” werden die nationale Einheit und Integrität, eine einheitliche Staatsstruktur, nationale Identität und Souveränität bezeichnet. Im Parteiprogramm wird nicht klar, ob die „nationalen Sensibilitäten“ und die nationale Kultur mit den Grundwerten der europäischen Integration im Widerspruch stehen oder nicht. Es gibt aber keine deutlichen Anzeichen, die auf eine Ablehnung der europäischen Integration zugrunde liegenden Werte hindeuten. Man kann davon ausgehen, dass auch hier keine explizite EU-pessimistische Haltung herrscht. Dennoch bedarf es einer weiteren Analyse. Letztlich wird die Interpretation der Äußerungen der Parteifiguren in verschiedenen Themenbereichen zeigen, welche Art des Euroskeptizismus bei der MHP zum Ausdruck kommt.

\section{Auswertung und Kategorisierung des Euroskeptizismus bei der MHP}

Dem Parteiprogramm kann man entnehmen, dass die MHP die europäische Integration und die EU-Mitgliedschaft explizit unterstützt. In diesem Abschnitt wird versucht zu definieren, welche Themenbereiche bei der MHP welche Art von Euroskeptizismus zum Ausdruck bringen. Es folgt die Zuordnung der Positionen der MHP zu den Arten des Euroskeptizismus. 
21 Eine nationalistische Haltung in der Zypernfrage ist nicht nur bei der MHP zu finden, aber im Vergleich zu anderen Parteien vertritt die MHP eine extrem nationalistische Position. Die Zypernfrage stellt bei der MHP also einen Themenbereich dar, in dem die EU heftig angegriffen wird. Diese Annahme wird in der folgenden Zitat unterstrichen:

„Die Europäische Union, die die Schwächen der AKP Regierung benutzt und die Zypernfrage zu einer "Guillotine“ für die Türkei gemacht hat, hat ihre diesbezüglichen Forderungen offen ausgedrückt und die Fristen dazu festgelegt. ${ }^{\text {“5 }}$ (MHP 2006: 6)

Hier deutet der Begriff „Guillotine“ mit seiner negativen Konnotation auf eine heftige Kritik der EU hin. Die Guillotine ist ein Fallbeil, das durch Enthauptung die Todesstrafe vollzog. Die Verwendung dieses Begriffs in diesem Zusammenhang weist demnach auf ein starkes Misstrauen gegenüber der EU hin.Die Forderung der EU, dass die Türkei konkrete Maßnahmen zur Normalisierung der Beziehung zu den griechischen Zyprioten einleitet, bedeutet nach Ansicht der MHP die politische Anerkennung. Nach Auffassung der MHP werde somit das türkische Veto aufgehoben, das eine Mitgliedschaft der griechischen Zyprioten in internationalen Organisationen verhindert. Es wurde laut MHP durch die letzten Entwicklungen noch einmal bestätigt, dass „die Zypern-Angelegenheit in dem mit der EU auf einer faulen Basis geführten Prozess der erste Knackpunkt sein wird. Es wurde klar, dass die als Zugunglück bezeichnete Trennung und Wegscheidung an der Haltestelle Zypern erfolgen wird“ (MHP 2006: 5).

Die Zypern-Politik der Europäischen Union wurde außerdem stark kritisiert, weil die Versprechen der EU gegenüber den Zyperntürken, Nordzypern finanziell zu unterstützen und die Handelsembargos und die Isolation aufzuheben, nicht realisiert wurden und die EU bis heute keine konkreten Schritte in diese Richtung unternommen hat. Die MHP behauptet, dass die ungerechte Ausgrenzung der zyprischen Türken in den Bereichen Politik, Wirtschaft, Transport, Tourismus und Sport bis heute anhält. Des Weiteren verachte die EU die Türkische Republik Nordzypern und erkenne nur die Zyperngriechen als einzige Vertreter der Insel an. Die MHP sieht also die Garantie der Sicherheit der Zyperntürken als eine unumgängliche politische Verantwortung und als eine nationale Aufgabe der Türkei. Die Rhetorik, die bei der Zypernfrage verwendet wird, deutet auf den EU-gegnerischen Charakter der Partei hin. In der Zypernfrage ist die Haltung der MHP nicht nur euroskeptisch, sondern EU-feindlich, denn die ideologische Positionierung der MHP (stark nationalistisch und xenophob), die auf einem negativen Fremdbild basiert, bestimmt das Handeln der MHP. Die hier verwendete Rhetorik reflektiert das große Misstrauen gegenüber der EU und der europäischen Integration wider, weshalb die Partei in die Kategorie der EU-Gegner einzuordnen ist.

Die MHP opponiert gegen die kulturellen Rechte der Minderheiten in der Türkei, weil eine Gewährung der Rechte ihrer Ansicht nach zur Auflösung der Türkei führen könnte. Sie betont auch, dass das Problem darin läge, dass in den Kopenhagener Kriterien eine weitgehende Gewährung von Minderheitenrechten von der Türkei gefordert werde. Die MHP interpretiert dies als eine potentielle Gefahr ethnischer Konflikte und eine Spaltung der Türkei. Der EU wird vorgeworfen den Friedensvertrag von Lausanne (1923) - auf dessen Grundlage die Türkei gegründet wurde - in den Vertrag von Sèvres umzuwandeln (MHP 2004: 16). Der MHP-Führer Devlet Bahçeli kritisierte die Forderung der EU die Todesstrafe abzuschaffen und mahnt die EU, mit Entschlossenheit gegen die terroristischen Organisationen zu kämpfen, die den Frieden 
und die Demokratie der Türkei bedrohen. Die MHP lässt sich von ihrer Befürwortung der Todesstrafe nicht abbringen, weil dies die Exekution des PKK-Führers Öcalan verhindern könnte.

„Dass der separatistische Terror den EU-Mitgliedschaftsprozess der Türkei als ein Mittel für seine politischen Ziele sieht und dass sich seine politische Strategie mit den Forderungen der EU an die Türkei deckt, sind Tatsachen, die niemand bestreiten kann." ${ }^{6}$ (Bahçeli 2004: 11)

Die MHP argumentiert, dass die Taktik der EU darin bestehe, das Nationalbewusstsein in der Türkei zu schwächen und in einem bestimmten Zeitraum den Nationalstaat in seiner einheitlichen Struktur aufzulösen. Die kulturellen Rechte für Minderheiten werden als erster Schritt dahin angesehen. Das pluralistische Gesellschaftsverständnis, das auf vielfachen Identitäten basiert, ist unvereinbar mit dem autoritären Staatsverständnis der MHP. Bei der MHP kann behauptet werden, dass sie bei diesem Thema gegen die Werte der EU opponiert. So ist die MHP eher als EU-Gegner zu klassifizieren.

In der Armenienfrage betont die MHP, dass die Anerkennung eines angeblichen Völkermords, der überhaupt nichts mit der $\mathrm{EU} \mathrm{zu}$ tun habe, nicht als eine Voraussetzung für die EU-Mitgliedschaft angesehen werden dürfe (Özdemir 2008: 14). Dass die Türkei ihre Grenze zu Armenien nicht geöffnet hat und sich keine Entwicklung zu einer nachbarschaftlichen Beziehung hin abzeichnet, wird im Fortschrittsbericht von 2006 kritisiert. Als eine Reaktion auf diese Kritik betitelte die MHP die EU als den „Anwalt von Armenien“. Außerdem kann nach Ansicht der MHP nicht akzeptiert werden, dass für die Lösung des Nagorni-Karabach Konflikts von der Türkei Beiträge verlangt wurden (MHP 2004: 51-52).Anhand der aufgeführten Beispiele ist die MHP als EU-skeptisch zu klassifizieren.

Der europäische Integrationsprozess wird mit der Vision eines „postmodernen Staates“ identifiziert, wobei der Schwerpunkt in der Bündelung von Souveränität, d.h. einer Verlagerung der Befugnisse des Staates auf die supranationalen Institutionen der EU. Darüber hinaus werden zunehmend externe Akteure, wie andere EU-Staaten, aber auch nichtstaatliche Akteure, in die Innenpolitik der Mitgliedstaaten involviert. Eine Besonderheit dieses postmodernen Staates ist die Anerkennung von multiplen Identitäten mit einer starken Betonung der Rechte der Minderheiten. Diese Vision von einem postmodernen Staat und die damit verbundenen Vorstellungen eines liberalen Internationalismus stehen in direktem Widerspruch $\mathrm{zu}$ den älteren, autoritären Versionen des Nationalismus, die eine einzige nationale Identität hervorheben. Diese Spannung ist in einem Land, in dem als historisches Erbe des Osmanischen Reiches und der Nation-Building-Erfahrung ein starkes Staatsverständnis herrscht, besonders zu spüren (Öniş 2003). Diese Spannungen wurden bei der Analyse des Euroskeptizismus der MHP deutlich erkennbar.

Im Parteiprogramm der MHP steht: „Die MHP akzeptiert prinzipiell die Vollmitgliedschaft in der EU, die mittlerweile eine einheitliche Staatspolitik vertritt.“ (MHP 2008). Diese Aussage steht im Widerspruch zu ihrem Handeln: Sie sieht die Freiheiten im Rahmen der EU-Normen als eine Bedrohung „der Einheit des Landes“ an, definiert die Gewährung von Rechten der religiösen Minderheiten als eine Auferstehung des Vertrages von Sèvres, wenn in den Mitgliedschaftsdokumenten von "kulturellen Rechten“ gesprochen wird. Außerdem beschuldigt sie die EU den Separatismus zu unterstützen und das „Recht auf Bildung in der Muttersprache“ als 
Wunsch, neben Türkisch auch Kurdisch zur amtlichen Schulsprache in den Grundschulen machen zu wollen darzustellen. Dass die MHP dem Begriff „Türkisch“ eine ethnische und diskriminierende Bedeutung gibt, wird aus ihrer Haltung zum Artikel 301 klar ersichtlich. Sie interpretiert die Änderung dieses Artikels als „die Freiheit das Türkentum zu beleidigen“ und startet Gegenkampagnen (MHP 2006: 3). Wenn auf den Widerspruch zwischen grundsätzlicher Zustimmung der EUMitgliedschaft und Ablehnung der Bedingungen einer Mitgliedschaft hingewiesen wird, erklären die MHP-Sprecher, dass sie nicht gegen eine Mitgliedschaft sind, sondern gegen eine Mitgliedschaftsbeziehung, die auf falsche Fundamente gesetzt wurde. Obwohl im Parteiprogramm die europäische Integration und die EU nicht explizit abgelehnt werden, kann in den Äußerungen der Parteifunktionäre heftige Kritik gegen die grundsätzlichen Ideen der europäischen Integration und die EU-Anforderungen festgestellt werden. Offizielle und reelle Positionen der MHP sind sehr unterschiedlich. Einige Begriffe, die für das Verständnis des Euroskeptizismus eine zentrale Rolle spielen, werden wiederholt verwendet. Die EU-Kommissare werden als Inspektor bezeichnet und die EU-Anforderungen zur Integration der Türkei werden als Falle oder Zwangsmaßnahmen charakterisiert. Dies deutet auf den EU-gegnerischen Charakter der Partei hin. Die Überzeugung von der ungerechten Behandlung durch die EU fällt mit einer Ablehnung der grundlegenden europäischen Ideen zusammen. Der einheitlichen nationalen Identität wird höchste Priorität beigemessen. Souveränitätstransfer an die EU-Institutionen und die Gewährung von Freiheiten bezüglich der Religion und Meinungsäußerung werden abgelehnt. Diese Beobachtungen zeigen, dass die MHP nach dem Modell von Kopecky und Mudde der Kategorie der EU-Gegner zugeordnet werden kann. Der Europadiskurs der MHP lässt sich mit grundlegenden europäischen Werten in der Demokratie, der Achtung des Individuums, dem Minderheitenschutz und der Meinungs- und Religionsfreiheit nicht vereinen.

Die MHP führt einen Europadiskurs, der mit einer andauernden scharfen Rhetorik und negativen Betitelungen der EU und ihren Akteuren verbunden ist. Die verbalen Äußerungen der MHP deuten darauf hin, dass sie den Souveränitätstransfer an die EUInstitutionen ablehnt. Die von der MHP gesprochene Sprache muss politikwissenschaftlich analysiert werden, damit die von der MHP bevorzugten „Denkbilder“ (Opp de Hipt 1987: 6) von der Europäischen Union und der Europäischen Integration erkannt und verstanden werden können. Um die Vorstellungen der MHP zu verstehen oder $\mathrm{zu}$ interpretieren, müssen die inneren Zusammenhänge des Gedankengebäudes der MHP nachvollziehbar sein (Vgl. Opp de Hipt 1987: 11) und sprachlichen Formen, in der die MHP die Europafrage bewertet, müssen genau analysiert werden. Die vorliegende Arbeit untersucht die Vorstellungen der MHP von der EU und der Europäischen Integration, soweit sie im Parteiprogramm, den Parteidokumenten und den Äußerungen des Parteivorsitzenden Devlet Bahçeli in den Medien nachzuweisen sind. Die MHP-Funktionäre setzen die Sprache in versteckter Weise als Mittel der Überredung und als Instrument der Manipulation ein und betreiben somit Propaganda (Vgl. Opp de Hipt 1987: 20). Emotionsgeladene Begriffe stehen im Zentrum der Reden der MHP-Funktionäre, die sich oft Metaphern bedienen um das Gesagte auszuschmücken und für den Zuhörer interessanter zu machen (zum Beispiel Guillotine als Metapher). Opp de Hipt bezieht sich auf Locke, der die bildhafte Rede kritisiert und glaubt, dass sie nur dazu da sei „to insinuate wrong ideas, move the 
passions, and thereby mislead the judgement" (Locke 1963: 288 zit. nach Opp de Hipt 1987: 49).

\title{
Analyse des Parteiprogramms der CHP
} die volle EU-Mitgliedschaft. Sie begründet diese Unterstützung mit der Modernisierungsperspektive des Parteigründers Mustafa Kemal Atatürk. Dies wird in folgender Passage deutlich:

\begin{abstract}
„Die CHP hat von je her die EU-Mitgliedschaft der Türkei unterstützt. Das Ziel der Türkei, ein volles Mitglied der EU zu werden, ist ein gesellschaftliches Wandlungsprojekt, das eine natürliche Fortführung der Revolution Mustafa Kemal Atatürks und seiner Vision von Modernisierung darstellt. Unsere Vorrausetzung für unsere Beziehungen zur EU ist eine gleichwertige, die Gründungswerte unserer Republik beachtende, ehrenhafte Vollmitgliedschaft. Die CHP kann eine von dieser Voraussetzung abweichende Möglichkeit nicht akzeptieren." ${ }^{7}$ (CHP 2008)
\end{abstract}

Neben dieser grundsätzlichen Unterstützung betont sie, dass die EU die Türkei formell mit den gleichen Kriterien wie die anderen Beitrittsländer bewerten muss. Die CHP akzeptiert nicht, dass der Türkei ein Sonderstatus im Gegensatz zu den übrigen Mitgliedern gegeben wird. Die Türkei akzeptiert alle Konditionen, die für eine Mitgliedschaft auch von den anderen Mitgliedern akzeptiert und umgesetzt wurden, unter anderem die Beachtung der Kopenhagener und Maastrichter Kriterien und die Annahme des EU-Rechts. Außerdem unterstützt sie die Beschleunigung und Vervollständigung des Reformprozesses. Die CHP stimmt einer privilegierten Partnerschaft, die von Angela Merkel und Nicolas Sarkozy oft vorgeschlagen wird, nicht zu. Hier wird ersichtlich, dass die Rhetorik einen stärkeren Charakter annimmt:

„Im Falle, dass die Politik einiger EU-Länder die Türkei aufgrund von geographischen und kulturellen Unterschieden aus der EU auszuschließen versucht und der Beschluss einer privilegierten Mitgliedschaft der Türkei zur offiziellen Position der EU wird, zur amtlichen Meinung der EU gemacht wird, so werden unsere gegebenen Zusagen, angeführt von der Zollunion, revidiert und die gemäß den Vorteilen unseres Landes erforderlichen Maßnahmen getroffen werden." ${ }^{\text {" }}$ (CHP 2008)

Die CHP besteht darauf, dass gegenüber den Ländern, die gegen eine Mitgliedschaft der Türkei sind, in angemessener Weise reagiert wird und Maßnahmen getroffen werden.Außerdem wird deutlich, dass die Einstellung der EU zur Zypernfrage die euroskeptische Haltung der CHP prägt. Auch kritisiert sie ist den Versuch willkürliche Konditionen, die im Widerspruch zum Friedensabkommen von Lausanne stehen, der Türkei aufzuzwingen:

"Die CHP verweigert eine Annäherung zur Lösung der Zypernfrage durch Druck und Zwang, um die griechische Seite zufrieden stellen zu können. Sie ist strikt gegen eine Annäherung, die die griechisch-zypriotische Regierung als den einzigen legitimen Staat, der Zypern vertritt, ansieht. Die CHP kann nicht akzeptieren, dass die griechisch-zypriotische Regierung in internationalen Organisationen und der Europäischen Union Gesamtzypern repräsentiert. “9 (CHP 2008)

Dass die EU-Mitgliedschaft der Türkei mit der Zypernfrage assoziiert wird und die Fortführung und das Ergebnis des Mitgliedschaftsprozesses an einseitige Kompromisse durch die Türkei gebunden wird, wird demnach nicht akzeptiert. Die CHP fordert grundsätzlich die Einhaltung des Lausanne-Vertrages. Die CHP unterstützt die Lösung der Zypernfrage nur unter der Voraussetzung, dass die erworbenen Rechte der 
Türkischen Republik Nordzypern und des nordzypriotischen Volkes berücksichtigt werden und dass eine Annäherung auf Basis der unabhängigen Gleichberechtigung beider Seiten der Insel stattfindet. Sie ist davon überzeugt, dass die Beziehungen zwischen beiden Ländern auf Zypern nur mit einer Annäherung in Fragen der Freundschaft, Solidarität, Zusammenarbeit und des permanenten Friedens normalisiert werden können. In diesem Rahmen unterstützt sie Friedensgespräche gemäß den Prinzipien der Gleichheit und des gegenseitigen Respekts.

Ein wichtiger Punkt im Parteiprogramm ist die Reaktion auf die EU-Staaten, die die Mitgliedschaft der Türkei aus geographischen und kulturellen Gründen ablehnen. Die öffentlichen Auftritte der Präsidenten dieser Staaten lösen bei den CHP-Funktionären starke Proteste aus. Der offene Charakter der Beitrittsverhandlungen ist ein anderer wichtiger Punkt, der bei der CHP auf Kritik stößt. Die CHP verlangt ein genaues Zieldatum für die volle EU-Mitgliedschaft der Türkei. Sie kritisiert die Beschränkungen der EU bezüglich der Landwirtschaftssubventionen, der Personenfreizügigkeit und der regionalen Entwicklung und fordert deren Aufhebung.

Im Parteiprogramm wird ersichtlich, dass die CHP die europäische Integration explizit unterstützt. Unter der Bedingung, dass die Aussagen der Parteifunktionäre nicht im Widerspruch zum Parteiprogramm stehen, kann sie als europhil klassifiziert werden. Im Parteiprogramm der CHP ist jedoch auch eine euroskeptische Einstellung erkennbar. Einerseits wird die europäische Integration unterstützt, andererseits wird betont, dass der aktuelle Stand der EU ihre eigenen Grundwerte nicht reflektiert. Um zu entscheiden, ob die CHP EU-Optimist oder EU-Pessimist ist, müssen die einzelnen Themenbereiche analysiert werden. Die Betrachtung einzelner Themenbereiche dient nicht nur dazu, die Gründe der Positionierung der CHP gegenüber der EU und der europäischen Integration zu klären, sondern auch einen Vergleich mit der Position der MHP zu ziehen.

\section{Auswertung und Kategorisierung des Euroskeptizismus bei der CHP}

In diesem Abschnitt wird definiert, in welchen Themenbereichen welche Art von Euroskeptizismus bei der CHP konkret zum Ausdruck kommt. Es folgt die Zuordnung der Positionen der CHP zu den Arten des Euroskeptizismus. Anhand des Parteiprogramms ist festzustellen, dass die CHP die europäische Integration explizit unterstützt.

Die Tatsache, dass die Zypernfrage die Hälfte des Gesamttextes im Parteiprogramm bezüglich der EU-Beziehungen umfasst, zeigt die Bedeutung des Themas im Europadiskurs der CHP. Die CHP ist überzeugt davon, dass die Türkei von Seiten der EU in der Zypernfrage ungerecht behandelt wird. Diese Überzeugung prägt den EU-Diskurs der CHP entscheidend.Die Kritikpunkte richten sich nicht gegen die grundlegenden Werte der europäischen Integration, sondern gegen die gegenwärtige Behandlung der Türkei durch die EU. Die CHP kritisierte die EU vor allem dafür, dass ihre Zypernpolitik parteiisch sei, da sie stark einseitig auf Griechenland ausgerichtet wäre und dass die Verträge von London und Zürich ignoriert würden (CHP 2007: 579). Die CHP kritisiert die Regierungspartei, weil diese den Annan-Plan zur Lösung der Zypernfrage unterstützen und der EU zugesagt hat, türkische Häfen und Flughäfen für zypriotische Flugzeuge und Schiffe zu öffnen (Öymen 2005: 533). Die CHP betont auch, dass die speziellen politischen Forderungen der Mitgliedstaaten keine Bedingung für den 
Beitritt der Türkei sein dürfen. Die Lösung der Zypernfrage sollte nicht als eine Voraussetzung für die Mitgliedschaft der Türkei gesehen werden. Eine ähnliche Praxis wurde im Verhandlungsprozess anderer Kandidatenländer nicht implementiert. Obwohl die CHP einen nationalistischen Diskurs führt und die Bewahrung des Status quo verteidigt, ist ihre Position bei der Zypernfrage eher EU-skeptisch als EUgegnerisch, denn die CHP betont in erster Linie, dass die Zypernfrage nicht als ein zusätzliches politisches Kriterium angesehen werden kann, weil es nicht zu den vom EU-Besitzstand abgeleiteten Anpassungsmaßnahmen gehört.

Die Gewährung der Rechtsstaatlichkeit, der Menschenrechte und des Minderheitenschutzes sind wichtige Elemente der europäischen Integration. Deniz Baykal ist der Meinung, dass die EU versuche, ein künstliches Minderheitenproblem zu schaffen. Seiner Ansicht nach herrscht in der EU das Verständnis, dass das Hervorheben verschiedener ethnischer Identitäten obligatorisch in einer Demokratie sei. Sobald es Menschenrechte und Demokratie gäbe, würde jeder seine Wurzeln suchen und eine Identität fordern. Dies nicht zu ermöglichen wäre ein Demokratiedefizit. Das ist nach Baykal ein völlig falsches Verständnis. Er betont, dass die Türkei ein Nationalstaat ist, der im Herzen eines Imperiums nach seiner Auflösung entstanden ist. Nach Baykal könne es also weder mit dem richtigen Demokratieverständnis noch mit der Fortschrittlichkeit einer Gesellschaft vereinbart werden, die Menschen dazu zu zwingen, die Identität einer ethnischen Minderheit anzunehmen oder anzuerkennen. Zudem wäre es falsch und beschämend, durch staatliche Zwangsmaßnahmen ethnische Identitäten unter Druck zu setzen. Baykal unterstreicht den Aspekt, dass niemand aufgrund seiner Wurzeln bevorzugt behandeln werden dürfe, schon gar nicht von einem Staat (Baykal 2004: 175-176). Die zurückhaltende Position in der CHP bei der Minderheitenfrage ist eher auf die inneren Widersprüche des Kemalismus zurückzuführen:

"The uneasy relationship between Kemalism and democracy becomes even more fragile when faced with identity politics -whether based on ethnic differences or on religious claims. Kemalism has traditionally viewed all quests for difference "not as natural components of a pluralistic democracy but as sources of instability and as threats to unity and progress." (Göle 1997: 84)

Man kann davon ausgehen, dass der Parteivorsitzende der sozialdemokratischen CHP die Minderheitenfrage im Kontext der Türkei beurteilt und die oben zitierte Sichtweise vertritt. Die Tatsache, dass die EU im Fortschrittsbericht 2004 Aleviten und Kurden als Minderheiten bezeichnet, wird daher als ein Problem der Sicherheit in der Türkei bewertet.Den offiziellen Dokumenten der CHP kann man eine EU-ähnliche Einstellung zum Minderheitenproblem entnehmen. Es kann festgehalten werden, dass die der EU zugrunde liegenden Werte in der Minderheitenfrage grundsätzlich akzeptiert werden, aber noch innerparteiliche Konflikte zu diesem Thema bestehen, die auf den Fraktionalismus innerhalb der Partei zurückzuführen sind. Deswegen ist es nicht einfach festzustellen, welche Art von Euroskeptizismus bei der CHP zum Ausdruck kommt. Es sollte auch zur Kenntnis genommen werden, dass das Konzept „Minorität“ neben seiner politischen und legalen Bedeutung eine soziologische Konnotation hat. Bei der soziologischen Definition heißt es, wenn eine Gruppe sich hinsichtlich der sozialen, politischen, kulturellen und ökonomischen Rechte gegenüber der Majorität benachteiligt behandelt fühlt, kann sie als eine Minorität bezeichnet werden. Wenn die Lage in der Türkei nach dieser Perspektive analysiert wird, können Kurden und Aleviten im Fortschrittsbericht der EU als Minoritäten bezeichnet werden. 

die Missachtung der Interessen der Türkei in Griechenland betont (Öymen 2004: 210), somit kommt die Ungerechtigkeitsrhetorik wieder zum Tragen. Grundsätzlich werden aber die Religionsfreiheit und die Rechte der Stiftungen religiöser Minderheiten unterstützt. Dieses Thema betreffend ist die CHP eher EU-skeptisch, obwohl man erwarten könnte, dass eine sozialdemokratische und linke Partei eine klarere Position zugunsten von Minderheitsstiftungen und Religionsfreiheit hat.

41 In der Armenierfrage ist eine euroskeptische Einstellung festzustellen. Die Meinung, dass die Armenierfrage nicht als Voraussetzung für den Beitritt angesehen werden darf, und den Historiker überlassen werden muss (Baykal 2004: 165; Öymen 2004: 211), deutet nicht auf einen Widerspruch zu den grundsätzlichen Ideen der europäischen Integration hin.Hinsichtlich des Artikels 301 kommt bei der CHP die nationalistische und konservative Variante des Kemalismus stärker zum Ausdruck. Die CHP behauptet, dass es in allen Ländern ähnliche Artikel gibt und dass diese nicht abgeschafft werden müssen (Öymen 2007: 13). Statt der Aufhebung des Artikels 301 fordert die CHP eine allgemeine Reform der Justiz. Die Sozialistische Internationale kritisiert die CHP hinsichtlich ihrer Position zum Artikel 301. Die Haltung der CHP dazu ist in erster Linie nicht vereinbar mit sozialdemokratischen Werten: Anklagen gegen Intellektuelle und Schriftsteller, die bei einem angeblichen Verstoß gegen diesen Artikel gegeben hat, dürfen in einem modernen und demokratischen Land nicht vorkommen. Auf der anderen Seite gibt es oft Reden von Parteifunktionären, die darauf bestehen, dass die Bestimmungen über Meinungsfreiheit und Menschenrechte in der Türkei auf keinster Weise den Bestimmungen europäischer Länder zurückstehen dürfen. Die Tatsache, dass mit Unterschriften von sechs Abgeordneten der CHP ein Antrag mit der Forderung gestellt wurde, Meinungen, die im Sinne einer Kritik geäußert werden, nicht in den Wirkungsbereich dieses Artikels fallen nicht als Straftat gelten zu lassen, kann wieder als positiver Schritt gewertet werden. Zusammenfassend kann festgestellt werden, dass die Position der CHP theoretisch nicht dem der europäischen Integration zugrunde liegenden Wert der Meinungsfreiheit widerspricht, die Haltung der CHP jedoch praktisch Konsequenzen hat, die zu diesem im Widerspruch stehen. Es kann aber behauptet werden, dass die CHP wegen der praktischen Konsequenzen des Artikels 301, nämlich der Beschränkung der Meinungsfreiheit, eher als EU-Gegner kategorisiert werden muss.

Ende 2004 entstanden externe Faktoren, die die Position der CHP gegenüber der EU durch den Türkeiskeptizismus der EU-Länder negativ beeinflusst haben: Die Ablehnung eines EU-Beitritts der Türkei bzw. der Vorschlag einer privilegierten Partnerschaft von Seiten des französischen Präsidenten Nicolas Sarkozy und der Bundeskanzlerin Angela Merkel, und die Tatsache dass die Aufnahmekapazität der EU als ein Beitrittskriterium geschaffen wurde, intensivierte das Vertrauensproblem der CHP gegenüber der EU. Die CHP interpretiert die dauerhaften Schutzmaßnahmen bezüglich der regionalen Entwicklung, Landwirtschaft und Personenfreizügigkeit als einen Verstoß gegen die EU-Normen. Hier wird der Kurs der EU als eine Abweichung von den grundlegenden Ideen der europäischen Integration bewertet (Baykal 2005: 269). Dieser Themenbereich ist entscheidend für die Einstellung der CHP gegenüber der EU und der europäischen Integration. Nach der Klassifizierung von Kopecky und Mudde kommt der EUskeptische Charakter der CHP zum Ausdruck. 

Euroskeptizismus der CHP. Das Agieren der EU, ihr Verhalten der Türkei gegenüber und die Diskurse, die sie hervorhebt, haben einen wesentlichen Einfluss auf die Debatte zwischen den Europhilen und den Europhoben in der Türkei und auf das Gleichgewicht zwischen beiden bei den innenpolitischen Verhandlungen im Beitrittsprozess (Kirişci 2003: 13). Wie Kopecky und Mudde hervorheben, kann das Verhalten der EU bei der CHP eine Transformation von der Kategorie der EU-Skeptiker zur Kategorie der EUEnthusiasten fördern. So eine Transformation kann wiederum zur Verwirklichung positiver Schritte seitens der Türkei führen. In diesem Fall ist es möglich über Iterationen und Interaktionen zu sprechen, die beide Seiten einander annähern und den Widerstand der Euroskeptiker schwächen oder die EU-Skeptiker in EUEnthusiasten verwandeln. Es muss also die Frage gestellt werden, ob die EU sich selbst ausreichend anstrengt, um die erforderlichen Anreize für die Entstehung eines Prozesses zu geben, der zu einer Verstärkung des politischen und institutionellen Wandels in der Türkei beiträgt. Die Tatsache, dass die Türkei historisch, politisch und kulturell als „der Andere“ charakterisiert wurde, führte dazu, dass eine ökonomische und institutionelle Europäisierung nicht ausreichend verwirklicht werden konnte. Die Kultur darf von der EU nicht als eine statische Entität verstanden werden, sondern als ein dynamischer Prozess (Giannakopoulos 2004: 59). Die Haltung der EU gegenüber der Türkei hat auch Auswirkungen auf die Fähigkeit der türkischen Regierung, verschiedene Akteure in der Innenpolitik für die Unterstützung der Reformen zu mobilisieren.

Es kann festgestellt werden, dass die CHP nach Kopeckys und Muddes Typologie eher als „EU-Skeptiker“ definiert werden kann. Es kommt im Allgemeinen eine positive Einstellung gegenüber der europäischen Integration zum Ausdruck, wobei bestimmte Themen betreffend eine negative Einstellung gegenüber der EU bemerkbar ist. Die grundlegende Unterstützung für europäische Normen wird betont, aber die EU als die aktuelle Verkörperung der europäischen Integration kritisiert. Die EU-Anforderungen werden in den genannten Themenbereichen kritisiert und die EU wird als eine Deviation von den grundlegenden Ideen der europäischen Integration bewertet. Hinsichtlich der europäischen Integration ist kein Widerspruch zwischen den Äußerungen der Parteifunktionäre und dem Parteiprogramm erkennbar. Es ist keine harte Rhetorik oder Angriff gegen die EU und ihre Akteure zu beobachten, die mit einer Ablehnung der der EU zugrunde liegenden Werte einhergehen. Der Euroskeptizismus bei der CHP basiert in erster Linie auf der Überzeugung davon, dass die Türkei von der EU ungerecht behandelt wird.

Zusammenfassend kann gesagt werden, dass der Euroskeptizismus der CHP von spezifischen Bedingungen der Kopenhagener Kriterien und von EU-Staaten, die aufgrund geographischer und kultureller Unterschiede gegen den Beitritt der Türkei sind, geprägt ist. Zwei Punkte der Kopenhagener Kriterien lösten bei der CHP besonders starke Proteste aus: Die Aufnahmefähigkeit der EU und der Artikel im Verhandlungsrahmen von Oktober 2005, der besagt, dass „die Verhandlungen ein offener Prozess sind, dessen Ergebnis nicht im Vorhinein garantiert werden könne“ (Kommission 2005: \&2). Dies ist nach Ansicht der CHP ein doppelter Maßstab der EU (CHP 2007). Obwohl allen Kandidaten gleiche Behandlung und die gleichen Aufnahmekriterien zugesichert wurden, waren nach Ansicht der CHP einige Mitglieder der EU nicht zur Aufnahme der Türkei als gleichberechtigtes Mitglied bereit, das mit 
der Idee eines multikulturellen Europas im Widerspruch stünde. Der Integrationsprozess der Türkei war demnach nicht von der Verwirklichung der Reformen abhängig. Es herrschte also eine große Skepsis darüber, ob die Türkei trotz Erfüllung aller Kriterien überhaupt aufgenommen werden würde.Der Euroskeptizismus der CHP kann auch als Reaktion auf den steigenden Türkeiskeptizismus Europas verstanden werden. Insgesamt lässt sich feststellen, dass die Überzeugung von der ungerechten Behandlung der Türkei durch die EU den Diskurs der CHP entscheidend prägt. Es gibt kein Zeichen dafür, dass der Souveränitätstransfer an die EUInstitutionen abgelehnt wird. Das Sèvres-Syndrom, das auf der Angst vor der Aufteilung der Türkei basiert, hat keinen Stellenwert im Diskurs der CHP. Die Betonung der nationalen Identität mit der Ablehnung der Veränderung des Artikels 301 stellt im gesamten Diskurs des Euroskeptizismus der CHP nur einen kleinen Stellenwert dar.

Viele liberale Beobachter argumentieren, dass innerhalb der Ideologie der CHP Elemente existieren, die zu dem Demokratieverständnis der EU im Widerspruch stehen. Die Agenden von fast allen türkischen politischen Parteien tragen nationalistische Züge. Ahmet Insel betont, dass dem historischen Kemalismus „ein ambivalentes Verhältnis zum Westen immanent ist" (Insel 2005: 62). Während sich die CHP-Führung einerseits tatsächlich darum bemüht, die sozialdemokratischen Parteien in Europa hinsichtlich der EU-Mitgliedschaft der Türkei zu überzeugen, „verkörpert die CHP alle inneren Widersprüche der offiziellen Staatsideologie der Türkei- des Nationalismus Atatürks, als deren Verteidiger sich die Partei auch versteht" (2005: 62). Dieser Widerspruch beinhaltet einerseits den starken Wunsch der westlichen Familie anzugehören und andererseits den Versuch den starken Status des Staates über die Gesellschaft zu erhalten. Die Haltung der CHP gegenüber der EU-Mitgliedschaft wird zum Teil dadurch erklärt, dass sie im Gegensatz zu anderen linken Parteien in Europa, auch wenn sie sich als eine linke Partei bezeichnet, nicht durch eine Bewegung von unten, sondern vielmehr durch die Elite von oben entstanden ist. Die CHP wurde von Debatten über den Multikulturalismus, den liberalen Internationalismus und die Politik des Dritten Weges, die die europäischen sozialdemokratischen Parteien stark prägte, nicht beeinflusst. Deswegen hat sie sich mit dem Staat und mit den republikanischen Werten identifiziert (Öniş 2007). Es kann argumentiert werden, dass der Konflikt vom kemalistischen Modernisierungsprojekt stammt, das die Verwestlichung trotz des Westens und trotz der Bürger voraussieht. Keyman und Öniş (2004: 173) behaupten, dass die staatszentrierte Politik in der Türkei eine Legitimitätskrise erlebt, weil im kulturellen und sozialen Leben Konflikte bezüglich ethnischer, kultureller oder religiöser Identität aufkommen. Sie betrachten eine Restrukturierung der Beziehungen des Staates zur Gesellschaft in eine liberale und demokratische Richtung als notwendig und vertreten die Ansicht, dass die theoretische und philosophische Basis des neuen Europas der postnationale Staat sei. In Anlehnung an Habermas (1998) erklären Keyman und Öniş (2004: 180-181), dass das neue Europa sich vom Nationalstaat zum postnationalen Staat umwandle. Diese Umstrukturierung beinhaltet das Aufkommen eines gewissermassen transnationalen Staates und resultiert einen Verlust an nationaler Souveränität. Die auf Sicherheit basierende Denkweise der CHP in der Türkei kann den postnationalistischen Staatsbegriff ihrer Ansicht nach nicht annehmen.

Aufgrund der qualitativen Analyse der Dokumente konnte in der vorliegenden Arbeit dargelegt werden, dass die CHP eher als EU-Skeptiker und die MHP eher als EU-Gegner klassifiziert werden können. Neben den Ausformungen des Euroskeptizismus beider 
Parteien ist die Frage der Kausalität ein wichtiger Aspekt. Welche kausalen Faktoren führen dazu, dass diese Parteien ihre jeweiligen Positionen gegenüber der EU und der europäischen Integration einnehmen? Wie schon zu Beginn der Arbeit erwähnt, soll die Frage geklärt werden, ob diese eher ideologisch-programmatische oder eher strategisch-taktische Gründe tragen? Im folgenden Kapitel wird dieser Frage nachgegangen, indem beide Parteien auf Basis der untersuchten Themenbereiche mit der Differenzmethode verglichen werden.

\section{VERGLEICH DES EUROSKEPTIZISMUS BEI DER MHP UND CHP} Stuart Mill (1874) gegenüber gestellt. Es wird nach Erklärungsfaktoren gesucht, die zu den verschiedenen Ausformungen des Euroskeptizismus bei der CHP und bei der MHP geführt haben. In Anlehnung an Mills Modell wird ein Merkmal als Erklärungsfaktor für die Varianz des Euroskeptizismus bestimmt, wenn es parallel zu den unterschiedlichen Ausformungen des Euroskeptizismus in den betrachtenden Parteien variiert. In der folgenden Untersuchung wird nach den unabhängigen Variablen, die eine Kovarianz mit der abhängigen Variablen bilden, gesucht (vgl. Blatter et al. 2007: 142).

In der Zypernfrage ist die Rhetorik der MHP der EU gegenüber ziemlich xenophob und stark nationalistisch. Es wird angedeutet, dass Griechenland -ein EU-Mitgliedstaatgeheime Pläne gegen die Türkei schmiede, wie im Begriff der Enosis deutlich wird (Bahçeli 2004: 8). Die ideologische Positionierung der MHP, die auf einem negativen Fremdbild basiert, bestimmt den Diskurs der MHP in der Zypernfrage. Bei der CHP kommt eher eine politische Haltung zum Ausdruck: Es wird nicht akzeptiert, dass die EU-Mitgliedschaft der Türkei mit der Zypernfrage assoziiert wird und die Fortführung und das Resultat des Mitgliedschaftsprozesses an einseitige Kompromisse von Seiten der Türkei gebunden wird. Außerdem verstärkt die Mitgliedschaft Südzyperns, das gegen den Annan-Plan stimmte, den Euroskeptizismus der CHP. Dies wird als eine ungerechte Behandlung durch die EU gesehen und stark kritisiert. Es muss aber einem weiteren wichtigen Aspekt Aufmerksamkeit geschenkt werden, nämlich dem innerparteilichen Fraktionalismus der CHP. Der stellvertretende Parteivorsitzende Onur Öymen sagte, dass die Haltung der CHP zum Thema Zypern sich nicht von derjenigen der MHP unterscheide (TBMM 2004). Diese Äußerung führte zu starken Diskussionen in der Partei, weil behauptet wurde, dass Öymen die CHP in eine rechte Richtung schieben würde. Die Kritiker in der CHP betonen, dass die CHP in Zypern eine Lösung anstrebe und mit der MHP nicht gleichgestellt werden dürfe. Der Istanbuler CHP Abgeordnete Halil Akyüz betont, dass die MHP gegen die EU-Mitgliedschaft sei und die Zypernfrage dafür benutze und die Positionen der CHP und der MHP überhaupt nicht übereinstimmen würden (TBMM 2004).Es kann festgestellt werden, dass die Zypernfrage u.a. den wichtigsten Kritikpunkt beider Parteien darstellt. Bezüglich der Zypernfrage ist die CHP eher als EU-Skeptiker und die MHP eher als EU-Gegner zu klassifizieren. Die Zypern-Frage kann als ein Erklärungsfaktor identifiziert werden, der bei den Parteien zu unterschiedlichen Ausformungen des Euroskeptizismus führt. Die Zypernfrage wird in der Tabelle 3 unter dem Punkt „Überzeugung von der ungerechten Behandlung der EU“ eingeordnet.

European Journal of Turkish Studies, Complete List 

das auf die Furcht vor einer ethnischen Spaltung der Türke hindeuteti. Die Türkei als Nationalstaat und ihre Einheit werden nach Ansicht der MHP in Frage gestellt. Es wird darauf hingewiesen, dass die PKK die EU für ihre eigenen Zwecke instrumentalisiert. Bei der CHP ist eine ambivalente Haltung zur Minderheitenfrage sichtbar, bei der die der EU zugrunde liegenden Werte jedoch nicht grundsätzlich verworfen werden. So ist die MHP eher als EU-Gegner zu klassifizieren. Eine klare Einordnung der CHP ist nicht möglich, weil Baykals Aussagen von der offiziellen Positionierung der CHP stark abweichen. Die Minderheitenfrage ist in der Tabelle 3 der Erklärungsvariablen SèvresSyndrom, zuzuordnen.

51 die MHP streng dagegen opponiert, dass die Türkei eine Geistlichen Schule auf der Insel Heybeliada öffnet, dem Fener Griechischen Patriarch ein politisches Status gewährt wird und dass er den Titel „ökumenisch“ auf offizieller Ebene führt (Bahçeli 2004: 17). Die Haltung der CHP ist eher pragmatisch-politisch. Die CHP ist als EU-Skeptiker, die MHP dagegen als EU-Gegner zu positionieren. Dieser Themenbereich wird in der Tabelle 3 unter kulturellen und religiösen Aspekten, die wiederum ein Erklärungsfaktor für die unterschiedlichen Ausformungen des Euroskeptizismus bei der CHP und MHP sind, eingeordnet. betonen, dass die Armenierfrage nicht als eine Voraussetzung für die EU-Mitgliedschaft angesehen werden darf. Beide Parteien können an dieser Stelle als EU-Skeptiker bezeichnet werden.

53 Bezüglich des Artikels 301 wird konstatiert, dass die MHP dem Begriff „Türkisch“ eine ethnische, rassistische Bedeutung beimisst. Dass die EU dies als Anlass zu ernster Besorgnis sieht und fordert, den Artikel 301 europäischen Standards anzupassen, hat nach Ansicht der MHP das Ziel, die Türkei zu einer Anerkennung des angeblichen Völkermords an den Armeniern zu zwingen. Diese Sichtweise ist mit dem Kriterium der Meinungsfreiheit nicht $\mathrm{zu}$ vereinbaren. Die CHP sieht in dem Artikel keine Beschränkung der Meinungsfreiheit und weist auf die Existenz ähnlicher Artikel in anderen europäischen Ländern hin. Auch die CHP muss in diesem Themenbereich der Kategorie der EU-Gegner zugeordnet werden, da der Artikel praktische Konsequenzen hatte, die die Meinungsfreiheit in der Türkei beschränkten. Dieser kausale Faktor kann deswegen ausgeschlossen werden, weil er nicht parallel zum Ergebnis variiert und gleiche Ausprägungen bei den beiden Parteien hat.

Der Grund für die euroskeptische Haltung der CHP liegt in erster Linie in externen Faktoren, die sich aus dem EU-Beitrittsprozess der Türkei ergeben: Zum einen wird dieser Prozess als einseitig ungerechte Behandlung durch die EU empfunden, zum anderen besteht ein Vertrauensproblem gegenüber der islamistischen Regierungspartei AKP. Die euroskeptische Haltung der MHP lässt sich mit dem SèvresSyndrom und dem Vorwurf, die EU würde die einheitliche Struktur der Türkei gefährden, begründen. Deswegen kann sie als EU-Gegner gesehen werden. Hinzu kommen ebenfalls das Vertrauensproblem gegenüber der Regierungspartei AKP und externe Faktoren.

Der erste Punkt bei der CHP besteht - wie bereits angedeutet - zum Teil aus einer Reaktion auf den steigenden "Turco-Skeptizismus“ in der Öffentlichkeit und in Parteien der EU-Länder (Yılmaz 2006).Die CHP konnte nicht verstehen, dass die 
Europäische Union aus Gruppen besteht, die verschiedene Interessen und Ideologien vertreten. Eine privilegierte Partnerschaft statt einer Vollmitgliedschaft, die Aufnahmekapazität der EU als ein Beitrittskriterium, andauernde Beschränkungen bezüglich Landwirtschaftssubventionen, Personenfreizügigkeit und regionaler Entwicklung und die Ergebnisoffenheit der Beitrittsverhandlungen sind in dieser Kategorie zu beobachten. Der damalige Kommissionspräsident Verheugen (2009) hat zugegeben, dass der größte Fehler der EU war, die Türkei nicht nach einer offenen, vertrauenswürdigen Linie behandelt $\mathrm{zu}$ haben. Verheugen spricht für eine klare Perspektive, die der Türkei angeboten werden solle. Der türkischen Regierung müsse deutlich gemacht werden, dass die EU-Mitgliedschaft von der Erfüllung der Kopenhagener Kriterien abhängt und die Türkei gemessen daran, ohne weitere Bedingungen in die EU aufgenommen werden würde. Dieser Aspekt prägt bei der MHP auch den Euroskeptizismus, aber die dahinter liegenden Denkbilder sind stark nationalistisch geprägt und unvereinbar mit den Werten der europäischen Integration. Das Argumentationsmuster der CHP ergänzt sich mit den grundlegenden Werten der europäischen Integration, ihre Argumente werden mit den Gesetzen der EU rational begründet. Die CHP bewertet das Verhalten der EU als eine Deviation von diesen Werten. Die Überzeugung von der ungerechten Behandlung seitens der EU kann als ein wichtiger Erklärungsfaktor bewertet werden, der zu unterschiedlichen Ausformungen des Euroskeptizismus bei der CHP und der MHP führt.

Die AKP wurde von der CHP beschuldigt, die EU-Mitgliedschaft als ein strategischtaktisches Ziel anzustreben mit der Absicht die Gesellschaft zu islamisieren und dies könne mit liberalem Gedankengut der EU leichter verfolgt werden. Laut CHP sei die AKP nicht laizistisch und könne deswegen die liberale Demokratie nicht verinnerlichen. Viele Forscher behaupten, dass die Situation der CHP als Oppositionspartei den Euroskeptizismus geprägt habe. Yılmaz (2006: 217) argumentiert, dass sich die Haltung der CHP von einem pro-europäischen Kurs allmählich zu einer politisch-euroskeptischen Position umwandelte, weil die konservative Regierungspartei AKP die Kontrolle bei dem Beitrittsprozess übernahm und die Wählerschaft bei Meinungsumfragen mit Misstrauen der CHP gegenüber standen. Da die CHP-Wähler mehrheitlich für einen EU-Beitritt sind, kann die euroskeptische Positionierung der CHP nicht mit strategisch-taktischen Gründen, wie beispielsweise die Absicht Wählerstimmen zu gewinnen, erklärt werden. Bei der MHP spielt das Vertrauensproblem auch eine starke, wenn nicht sogar eine bestimmende Rolle für ihren Euroskeptizismus. Die Begründung des Euroskeptizismus mit der Absicht Wählerstimmen zu gewinnen, ist bei der MHP aus den gleichen Gründen wie bei der CHP unglaubwürdig. Das Vertrauensproblem gegenüber der AKP und das Motiv der Stimmenmaximierung können als Erklärungsfaktoren ausgeschlossen werden, da sie zu gleichen Ausformungen des Euroskeptizismus bei den Parteien führen.

Das Sèvres-Syndrom spielt eine entscheidende Rolle im Diskurs der MHP. Die CHP akzeptiert einen Souveränitätstransfer unter der Bedingung, dass alle Beitrittskandidaten gleich behandelt werden. Der Diskurs der CHP basiert hauptsächlich an der Überzeugung von der ungerechten Behandlung der Türkei, die als eine Abweichung von den grundlegenden Ideen der europäischen Integration bewertet wird. Auch die MHP weist auf die ungerechte Behandlung der Türkei durch die EU hin, macht aber dabei von einer aggressiven Rhetorik und Begründungen Gebrauch, die explizit mit den der EU zugrunde liegenden Werten im Widerspruch stehen. Die EU wird oft als ein „Christenclub“ bezeichnet, der eine andere kulturelle und religiöse 
Grundlage hat und deswegen unvereinbar mit den kulturellen Werten der Türkei sei. Die Gewährung einer Ökumene an das Patriarchat und die Öffnung der Geistlichen Schule werden extrem entschieden abgelehnt. Die CHP hat in kultureller Dimension eine pro-europäische Orientierung, die oft in den Reden der Parteifunktionäre deutlich wird. In Tabelle 3 wird deutlich, dass bestimmte kausale Faktoren (Stimmenmaximierung, Vertrauensproblem gegenüber der AKP, Abschaffung des Artikels 301 und die Armenierfrage) ausgeschlossen werden und die Erklärungsvariablen (Die Überzeugung von der ungerechten Behandlung durch die EU, Salienz der Sèvres-Syndroms, Souveränitätstransfer an die EU-Institutionen, kulturelle und religiöse Unterschiede) identifiziert werden. 
Tabelle 3: Vergleich des Euroskeptizismus bei der CHP und bei der MHP

\begin{tabular}{|c|c|c|}
\hline & DIE MHP & DIE CHP \\
\hline $\begin{array}{l}\text { Zum Euroskeptizismus beitragende } \\
\text { Faktoren im türkischen Kontext als } \\
\text { unabhängige Variablen: }\end{array}$ & & \\
\hline \multicolumn{3}{|l|}{ Ausgeschiedene Kausale Faktoren } \\
\hline Stimmenmaximierung & Nein & Nein \\
\hline Vertrauensproblem mit der AKP & $\mathrm{Ja}$ & $\mathrm{Ja}$ \\
\hline Abschaffung des Artikels 301 & $\mathrm{Ja}$ & $\mathrm{Ja}$ \\
\hline $\begin{array}{l}\text { Das Beharren der EU auf der Anerkennung } \\
\text { eines Völkermords an Armenien seitens } \\
\text { der Türkei }\end{array}$ & $\mathrm{Ja}$ & $\mathrm{Ja}$ \\
\hline \multicolumn{3}{|l|}{ Erklärungsvariablen } \\
\hline $\begin{array}{l}\text { Die Überzeugung von der ungerechten } \\
\text { Behandlung durch die EU } \\
\left(\text { als externe Faktoren }^{1} \text { ) }\right.\end{array}$ & 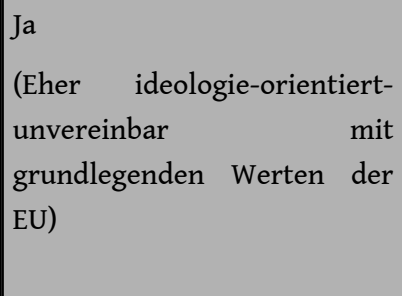 & $\begin{array}{l}\text { Ja } \\
\text { (Eher politik- } \\
\text { orientiert } \\
\text {-vereinbar mit } \\
\text { grundlegenden } \\
\text { Werten der EU) }\end{array}$ \\
\hline $\begin{array}{l}\text { Salienz des Sévres-Syndroms } \\
\text { (Bedrohung der nationalen Einheit- } \\
\text { Separatismusangst) }\end{array}$ & Hoch & Niedrig \\
\hline $\begin{array}{l}\text { Souveränitätstransfer an die EU- } \\
\text { Institutionen }\end{array}$ & Nein & $\mathrm{Ja}$ \\
\hline Kulturelle und Religiöse Unterschiede & $\mathrm{Ja}$ & Nein \\
\hline Ergebnis & EU-GEGNER & EU-SKEPTIKER \\
\hline
\end{tabular}

1. Zypernfrage, Privilegierte Partnerschaft, Offenheit der Beitrittsverhandlungen, Äußerungen der Präsidenten derEU-Mitgliedsländer, Beschränkungen der EU bezüglich der Landwirtschaftssubventionen, Personenfreizügigkeit und der regionalen Entwicklung, Aufnahmefähigkeit der EU als spezielle Bedingung für die Türkei

Die Wähler beider Parteien unterstützen die EU-Mitgliedschaft mehrheitlich. Außerdem ist die Salienz der Europafrage in den Wahlen ziemlich gering. Seit 2004 ist in der türkischen Öffentlichkeit ein Rückwärtstrend in der Befürwortung der EUMitgliedschaft der Türkei zu beobachten. Eurobarometer-Umfragen zufolge fiel der Anteil der Türken, die die Mitgliedschaft in der EU als "gute Sache" beurteilten, von 71 Prozent im Jahre 2004 auf 54 Prozent im Jahre 2006 und auf 49 Prozent im Jahre 2007 
(Eurobarometer 2008: 23). Trotz dieses negativen Trends sollte angemerkt werden, dass mindestens die Hälfte der türkischen Öffentlichkeit die EU in dem besagten Zeitraum als positiv bewertete. Deswegen ist es problematisch zu behaupten, dass diese Parteien nur für die Stimmenmaximierung eine bedingt oder grundsätzlich ablehnende Position gegenüber der EU vertreten. Die strategisch-taktischen Ziele sind nicht unbedingt ausschlaggebend für die Ausformungen des Euroskeptizismus, die bei den beiden Parteien zum Ausdruck kommen. Bei beiden Parteien scheinen in den untersuchten Themenbereichen eher breitere ideologische Profile und Werte im Vordergrund $\mathrm{zu}$ stehen. Kopecky und Mudde (2002: 319-321) haben festgestellt, dass extrem rechte Parteien oder kommunistische Parteien (am Rande des politischen Spektrums stehende Parteien) europhob sind. Die MHP vertritt eine stark nationalistische Ideologie, die mit den grundlegenden Werten der EU unvereinbar ist und somit europhob erklärt werden kann. Die sozialdemokratische CHP, die wegen der inneren Widersprüche des Kemalismus in manchen Themenbereichen, wie der Minderheitenfrage, eine ambivalente Haltung aufzeigt, ist optimistisch gegenüber der europäischen Integration und ist demnach EU-skeptisch. Die Ausformungen des Euroskeptizismus sind vereinbar mit ihrer jeweiligen Parteifamilie. Es kann argumentiert werden, dass bei diesen Parteien die Haltung gegenüber der europäischen Integration von ihrer jeweiligen Ideologie bestimmt wird. Der Überzeugung von der ungerechten Behandlung seitens der EU ist weder als strategisch noch als ideologisch zu beurteilen und kommt als ein externer Faktor zum Ausdruck, der einen besonders starken Stellenwert bei der CHP hat. Das Vertrauensproblem gegenüber der AKP muss vielmehr auf einer ideologischen Basis interpretiert werden und ist auf den Konflikt zwischen Islamismus und Kemalismus zurückzuführen. In der Türkei existiert also der Euroskeptizismus nicht nur bei zentripetalen sondern auch bei den zentrifugalen Kräften. Ideologie ist die erklärende Variable im Kontext der Haltungen der Parteien gegenüber Europa. In diesem Zusammenhang wird die These von Kopecky und Mudde, die auf eine Kongruenz zwischen bestimmten Ideologien und der Wahrscheinlichkeit zur Einnahme euroskeptischer Positionen hindeutet, bei der CHP und der MHP bestätigt.

\section{FAZIT}

Seit dem Helsinki Gipfel 1999 vollzieht sich in der Türkei ein grundlegender Wertewechsel. In zahlreichen Gesetzespaketen sind tiefgehende Reformen erreicht worden. Die Träger des Beitrittsprozesses in der Türkei sind politische Parteien, welche die Europäische Union zunehmend skeptisch beurteilen. Der bis jetzt in der Türkei wenig diskutierte parteibasierte Euroskeptizismus der CHP und der MHP könnte den Fortgang des Integrationsprozesses bremsen. Um dieses Problem näher zu beleuchten, wurden in der vorliegenden Arbeit in der sozialwissenschaftlichen Literatur zum Euroskeptizismus existierende Konzepte betrachtet. Dieses Phänomen wurde bei der CHP und der MHP auf der Basis der von Kopecky und Mudde konstruierten Typologie untersucht, die zwischen einer diffusen und spezifischen Unterstützung unterscheidet. Die CHP spielt in diesem Prozess die Rolle „der unzufriedenen Demokraten“, die sich von der EU betrogen fühlen, die aber auch Widersprüche des Kemalismus zu bewältigen haben, während die MHP als Vertreter einer extremen nationalistischen Weltanschauung angesehen werden kann. 
Das imaginäre Europa ist sowohl im öffentlichen Diskurs als auch im Diskurs der politischen, militärischen und bürokratischen Eliten der Türkei ein permanenter Referenzpunkt. Das „europäische Andere“ wurde in der türkischen Geschichte sowohl als eine Gefahr als auch ein Wunschobjekt für die türkische Identität betrachtet. Im Prozess der Definition der türkischen nationalen Identität im späten neunzehnten Jahrhundert bis heute wurde Europa entweder als ein Modell zu Nachahmung begrüsst oder als eine Bedrohung der eigenen nationalen Werte abgelehnt (vgl. Ahiska 2003, Küçük 2008). Im Laufe der Geschichte kann ein Misstrauen der Türkei gegenüber der EU beobachtet werden. Dieses Misstrauen scheint sich auch bei der CHP und bei der MHP in verschiedenen Ausformungen widerzuspiegeln, denn politische Parteien können von der Gesellschaft, in der sie agieren, nicht getrennt betrachtet werden. Politik ist ein dynamischer Prozess, in dem sich die Gleichgewichte verändern und die Hindernisse für einen EU-Beitritt der Türkei beseitigt können. Dies hängt sowohl von den positiven Einstellungen der europäischen Akteure gegenüber der Türkei, als auch von denen der türkischen Akteure ab. Je positiver die EU der Türkei gegenüber tritt, desto fähiger ist die türkische Seite die Widerstände und Hindernisse auf dem Weg zu einer besseren EU-Türkei-Beziehung zu überwinden. Gegenseitiges Vertrauen ist der erste Schritt hin zu einer gesunden Beziehung, die auf die Vollmitgliedschaft der Türkei in der EU abzielt.

\section{BIBLIOGRAPHIE}

Ahiska, Meltem (2003) "Occidentalism: The Historical Fantasy of the Modern", in The South Atlantic Quarterly. vol. 102, spring 2003, pp. 351-379.

Bahçeli, Devlet (2004) “Presseerklärungen”, in MHP Haklı Çıkmıştır (Die MHP hat Recht), Ankara, MHP Verlag, pp. 7-75.

Baykal, Deniz (2004) "Pressemitteilungen der Parteivorsitzenden der CHP über den Fortschrittsbericht von 2004. 8.-19. Oktober 2004", in AB’ye Tam Üyeliğe Evet, Özel Statüye Hayır (Ja zur Vollmitgliedschaft, Nein zur Privilegierten Partnerschaft), Ankara, CHP Verlag, 2007, pp. 152-180.

Baykal, Deniz (2005) “Ansprachen des Parteisvorsitzenden der CHP bezüglich des Entscheids des Europäischen Rates am 17. Dezember 2004 und die weiteren Entwicklungen nach diesem Datum in der Generalversammlung der Partei, 25 Mai 2005", in AB'ye Tam Üyeliğe Evet, Özel Statüye Hayır (Ja zur Vollmitgliedschaft, Nein zur Privilegierten Partnerschaft), Ankara, CHP Verlag, 2007, pp. 268-273.

Blatter, Joachim K.; Janning, Frank; Wagemann, Claudius (2007) Qualitative Politikanalyse. Eine Einführung in Forschungsansätze und Methoden, Wiesbaden, VSVerlag für Sozialwissenschaften.

CHP (2007), AB Bülteni: AB’ye Tam Üyeliğe Evet, Özel Statüye Hayır (Ja zur Vollmitgliedschaft, Nein zur Privilegierten Partnerschaft), Ankara, CHP Verlag.

CHP (2008) Parteiprogramm, URL: http://www.chp.org.tr/Dosyalar.aspx?FileCatID=5

[14.04.2009] 
Easton, David (1965) A Framework for Political Analysis, Englewood Cliffs, NJ, Prentice-Hall.

Eder, Mine (2004) "Populism as a barrier to integration with the EU. Rethinking the Copenhagen criteria", in Uğur, Mehmet; Canefe, Nergis(Hrsg) Turkey and European Integration. Accession Prospects and issues, London, Routledge, 2004, pp. 49-74.

Eurobarometer 69 (2008) Public Opinion in the European Union, National Report: Executive summary: Turkey, European Commission, Spring 2008.

Europäische Kommission (2005) “Türkei Verhandlungsrahmen”, 2005.

Giannakopoulos, Angelos (2005) Europäische Integration und kulturelle Denk- und Wahrnehmungsmuster. Kulturelle Aspekte des EU-Erweiterungsprozesses anhand des Verhältnisses Europäische Union - Türkei, Forschungsprojekt, Universität Konstanz. Geisteswissenschaftliche Sektion.

Göle, Nilüfer (1997) "The Quest for the Islamic Self Within the Context of Modernity" in Bozdogan, Sibel; Kasaba, Resat, Rethinking Modernity and National Identity in Turkey, Seattle, University of Washington Press, pp. 81-94.

Habermas, Jürgen (1998) Die postnationale Konstellation: Politische Essays, Suhrkamp.

Insel, Ahmet (2005) Demokratisierungsdiskurse der türkischen Parlamentarier bezüglich der EUBeitrittsoption der Türkei, Teiluntersuchung im Rahmen des VW-Forschungsprojekts: Europäische Integration und kulturelle Denk- und Wahrnehmungsmuster, Kulturelle Aspekte des EUErweiterungsprozesses anhand der Beziehungen EU-Türkei.

Keyman, F.; Öniş; Z. (2004) "Helsinki, Copenhagen and beyond. Challenges to the New Europe and the Turkish State" in Uğur, Mehmet; Canefe, Nergis(Hrsg) Turkey and European Integration. Accession Prospects and issues, London,Routledge, 2004, pp. 173-194.

Kirişci, Kemal (2003) "Turkey and the European Union: The Domestic Politics of Negotiating PreAccession”, in Macalester International, vol. 15, Spring 2005.

Kopecky, P., Mudde, C. (2002) “The Two Sides of Euroscepticism: Party Positions on European Integration in East Central Europe”, in European Union Politics, 3 (3), pp. 297-326.

Küçük, Bülent (2008) Die Türkei und das andere Europa. Phantasmen der Identität im Beitrittsdiskurs. Bielefeld, Transcript Verlag.

Marks, G., Hooghe, L., Wilson, C. (2002) "Does Left/Right Structure Party Position on European Integration?", inComparative Political Studies, 35 (8), pp. 965-989.

Mayring, Philipp (2008) Qualitative Inhaltsanalyse: Grundlagen und Techniken, Weinheim und Basel, Beltz Verlag.

MHP (2004) MHP Haklı Çıkmıştır (Die MHP hat Recht), Ankara, MHP Verlag, URL: http:// www.mhp.org.tr/dokumanlar_yayinlar.php [12.06.2009]

MHP (2005) İşte! AKP’nin Avrupa Birliği Yol Haritası: Çıkmaz Sokak' (2005)-(AKPs Fahrplan zur EU: „Eine Sackgasse“), MHP Forschungszentrum, Ankara, MHP Verlag, URL: http://www.mhp.org.tr/ dokumanlar_yayinlar.php [01.08.2009]

MHP (2006) “2006 Yılı İlerleme Raporu: Siyasi Kriterler Çerçevesinde Talep ve Dayatmalar (Der Fortschrittsbericht von 2006: Forderungen und Zwangsmassnahmen im Rahmen der Politischen Kriterien)", MHP Forschungszentrum, Ankara, MHP Verlag, URL: http://www.mhp.org.tr/ dokumanlar_yayinlar.php [01.08.2009]

MHP (2008) Parteiprogramm, URL: http://www.mhp.org.tr/mhp_parti_programi.php[11.07.2009] 
Mill, John S. (1874) A System of Logic. Ratiocinative and Inductive: Being a connected view of the principles of ecidence and the Methods of scientific investigation, NewYork, Harper \& Brothers.

Opp de Hipt, Manfred (1987) Denkbilder in der Politik, Opladen, Westdeutscher Verlag.

Öniş, Ziya (2003) "Domestic Politics, International Norms and Challenges to the State: Turkey-EU Relations in the Post Helsinki Era”, inTurkish Studies, 2003, 4 (1). In Ali Carkoglu and Barry Rubin (Hrsg.), Turkey and the European Union, London and Portland, Frank Cass, 2003, pp. 16-48.

Öniş, Ziya (2007) “Conservative Globalists versus Defensive Nationalists: Political Parties and Paradoxes of Europeanization in Turkey", injournal of Southern Europe and the Balkans, vol. 9, $\mathrm{n}^{\circ}$ 3,http://www.portal.ku.edu.tr/ zonis/ONIS-EKEM-NOV\%202006.pdf [12.03.2009]

Öymen, Onur (2004) "Rede des Stellvertretenden Parteivorsitzenden der CHP im Fernsehprogramm „Mots Croises“ im zweiten Kanal des französischen Fernsehens”, in AB'ye Tam Üyeliğe Evet, Özel Statüye Hayır (Ja zur Vollmitgliedschaft, Nein zur Privilegierten Partnerschaft), Ankara, CHP Verlag, 2007, pp. 210-213.

Öymen, Onur (2005) "Pressekonferenz des stellvertretenden Parteivorsitzenden der CHP vom 19. Oktober 2005”, in AB'ye Tam Üyeliğe Evet, Özel Statüye Hayır (Ja zur Vollmitgliedschaft, Nein zur Privilegierten Partnerschaft), Ankara, CHP Verlag, 2007, pp. 531-536.

Öymen, Onur (2007) Rede des stellvertretenden Parteivorsitzenden der CHP bei seiner Ansprache auf einer Konferenz an der Fakultät für Politikwissenschaften der Universität Ankara am 19. Februar 2007.

Özdemir, Ali Ulvi (2008) Türkiye'de Avrupa Birliği Karşıtlğının Tarihi (Die Geschichte der Opposition gegenüber der Europäischen Union in der Türkei), Ankara, Ilksan Verlag.

Sitter, Nick (2001) “The Politics of Opposition and European Integration in Scandinavia: Is Eurosceptism a Government-Opposition Dynamic?", in West European Politics, 24, 2001, pp. 22-39.

Sitter, Nick (2002) Opposing Europe: Euro-scepticism, Opposition and Party Competition, SEI Working Paper No. 56 / OERN Paper No. 9, Brighton, University of Sussex/Sussex European Institute, URL: http://www.sussex.ac.uk/sei/documents/wp56.pdf [18.04.2009]

Taggart, Paul (1998) “A Touchstone of Dissent: Eurosceptism in Contemporary Western European Party Systems”, in European Journal of Political Research, 33, pp. 363-388.

Taggart, P., Szczerbiak, A. (2001) "Parties, Positions and Europe: Euroscepticism in the EU Candidate States of Central and Eastern Europe", inOpposing Europe Research Network Working Paper, $\mathrm{n}^{\circ}$ 2, Sussex European Institute, URL: http://www.sussex.ac.uk/sei/documents/wp46.pdf [12.08.2009]

Taggart, P., Szczerbiak, A. (2003) Theorising Party-Based Euroscepticism: Problems of Definition, Measurement and Causality, SEI Working Paper nº6, Oktober 2003.

TBMM (2004) Türkiye Büyük Millet Meclisi Genel Kurul Tutanagi ( Grosse Nationalversammlung der Türkei Hauptversammlungsprotokoll) 22.Periode, 3.Legislationsjahr, 8.Sitzung. 20 Dezember 2004 (Besprechungen über den Entscheid des Europäischen Rats am 17. Dezember 2004 über die Aufnahme von Beitrittsverhandlungen mit der Türkei zum 03.10.2005)

Yılmaz, Hakan (2006) "Euroskeptizismus in der Türkei- Parteien, Eliten und öffentliche Meinung, 1995-2006", in Gabriele Clemens (Hg.) Die Türkei und Europa, Berlin, LIT Verlag, pp. 215-245.

Verheugen, Günter (2009) “Verheugen'den AB Ülkelerine Türkiye Eleştirisi (Verheugens Kritik zu EULänder in Bezug auf die Türkei) ”, in Radikal, 02/03/2009. 


\section{NOTES}

1. Dieser Begriff wird oft in den Monographien der türkischen Sozialwissenschaftler verwendet und deutet auf den tiefgreifenden Wandel in der türkischen Politik nach der Anerkennung als Beitrittskandidat auf dem Helsinki-Gipfel im Jahr 1999 hin.

2. Die pro-kurdische DTP wurde im Jahr 2005 gegründet. Sie war die Nachfolgerin der HADEP, die im Jahr 1994 gegründet und im Jahr 2003 verboten wurde, und der DEHAP, die sich im Jahr 2005 angesichts eines Verbotsverfahrens selber auslöste. Die DTP wurde am 11. Dezember 2009 durch das Verfassungsgericht verboten und gründete sich dann als BDP neu.

3. Anmerkungen zur Tabelle 2 (Wichtige Faktoren zur Analyse des Euroskeptizismus):

Zur Rhetorik: Im Laufe dieser Einordnung von Textpassagen wird deutlich, welche Begriffe wiederholt verwendet werden und für das jeweils transportierte Verständnis von Euroskeptizismus zentral sind. Solche Begriffe können als Schlüsselbegriffe bezeichnet werden. Da die Art der Verwendung solcher Schlüsselbegriffe die Entwicklung des Diskurses wesentlich prägt, wird ihnen in der Analyse besondere Aufmerksamkeit geschenkt. Diese Schlüsselbegriffe sind wichtige Zeichen für die reale Position der Parteien zur EU. Bei andauernder harter Rhetorik und Anfeindungen gegenüber der EU und ihren Vertreter verbunden mit einer Ablehnung der der EU zugrunde liegenden Werte, wird die Partei der Kategorie der EU-Gegner zugeordnet (Bezeichnung der EU-Kommissare als Inspektor und der EU-Anforderungen als Falle oder Zwangsmaßnahmen gegen die Türkei).

Die Überzeugung von der ungerechten Behandlung der Türkei von Seiten der EU in bestimmten Themenbereichen (wie am Begriff „doppelter Maßstab“ deutlich wird), deutet eher auf den EUskeptischen Charakter der Partei hin, auch wenn die Europa zugrunde liegenden Werte allgemein akzeptiert werden. EU-skeptische Parteien bewerten die ungerechte Behandlung seitens der EU als eine Deviation von den grundlegenden Ideen der europäischen Integration. Wenn die Überzeugung von der ungerechten Behandlung durch die EU mit einer Ablehnung der grundlegenden europäischen Ideen zusammenfällt, dann zeigt dies, dass die Partei ein EU-Gegner ist. Die Überzeugung von der ungerechten Behandlung durch die EU wird im türkischen Diskurs in folgenden Themen erkennbar: Keine finanzielle Hilfe durch die EU bis 2014, privilegierte Partnerschaft, der offene Charakter der Beitrittsverhandlungen, der Druck der EU bezüglich der Erfüllung der Forderungen Griechenlands, die Anerkennung der Griechen als legitime Vertreter Zyperns, die Anerkennung Süd-Zyperns (das Protokoll zur Anpassung des Ankara-Vertrags), die Anerkennung des Völkermords an den Armeniern, Beschränkungen der EU bezüglich der Landwirtschaftssubventionen, Personenfreizügigkeit und die regionale Entwicklung, Aufnahmefähigkeit der EU als spezielle Bedingung für die Türkei. Die Betonung der ungerechten Behandlung durch die EU wird oft deutlich anhand der Reaktionen auf Äußerungen von Präsidenten der EU-Mitgliedsländer.

Die Ablehnung des Souveränitätstransfers an die EU deutet darauf hin, dass die Partei EU-Gegner ist, denn die europäische Integration verlangt die Verlagerung eines Teils der nationalen Souveränität auf die EU-Institutionen. Folgende Indikatoren deuten auf eine Ablehnung des Souveränitätstransfers hin: Sèvres-Syndrom, die Veränderung der einheitlichen Struktur des Staates, die Schwächung der nationalen Unabhängigkeit, das Ende des türkischen Staats, Separatismusangst.

Hohe Priorität der nationalen Identität und Religion deuten darauf hin, dass die Partei EUGegner ist. Bezeichnung der EU als Christen-Club, die Ablehnung der Gewährung einer Ökumene an das Patriarchat und der Eröffnung der Geistlichen-Schule sind Indikatoren für die Bedeutung der Religion. Welche Priorität der nationalen Identität bei einer Partei gegeben wird, kann aus folgenden Indikatoren herausgelesen werden: Die Ablehnung der Veränderung des Artikels 301, die Ablehnung der Bildung in einer anderen Sprache, Überzeugung von der Unterstützung der 
PKK durch manche EU-Länder, Überzeugung von der Spaltung der Türkei durch ethnische Differenzierung und von der Erosion der moralischen Werte durch die EU.

In Anlehnung an Kitschelts Ideen kann argumentiert werden, dass die Betonung von kosmopolitischen und sozialliberalen Werten eher den EU-enthusiastischen Charakter einer Partei aufzeigt, und eine nationalistische, autoritäre Haltung eher den EU-gegnerischen oder den EU-skeptischen Charakter einer Partei ausdrückt.

Die pro-europäische Orientierung der Parteien in kultureller Dimension (die Art in der zu westlichen Werten Stellung bezogen wird) deutet auf den EU-enthusiastischen Charakter einer Partei hin.

4. „Türk Milleti için vazgeçilemez nitelik taşıyan unsurlar; millî kültürümüze, toprak bütünlüğümüze ve üniter devlet yapımıza olan saygı ve bağlılıktır. Avrupa Birliğine dahil ülkelerin millî duyarlılıklarımıza gösterecekleri saygı, bu ülkelerle olan ilişkilerimizin geleceğini belirleyecek temel kriterleri oluşturacaktır." (MHP 2008)

5. "AKP hükümetinin zaafını kullanarak Kıbrıs sorununu Türkiye'nin karşısına bir "giyotin" olarak çıkaran Avrupa Birliği, bu konudaki dayatmalarını açıç̧a ortaya koymuş ve bunun vadelerini belirlemiştir." (MHP 2006: 6)

6. “Bölücü terörün Türkiye'nin $A B$ üyelik sürecini kendi siyasî hedeflerine hizmet edecek bir araç olarak gördüğü ve siyasî stratejisinin AB'nin Türkiye'den talepleriyle örtüştüğü, kimsenin inkâr edemeyeceği gerçeklerdir.” (Bahçeli 2004: 11)

7. „CHP başından beri Türkiye'nin AB üyeliğini desteklemektedir. Türkiye'nin AB'ye tam üyelik hedefi, Mustafa Kemal Atatürk'ün çağdaşlaşma devriminin, modernleşme vizyonunun doğal uzantısı olan bir toplumsal değişim projesidir. AB ile ilişkilerimizde koşulumuz; eşit koşullu, Cumhuriyetimizin kuruluş değerlerine saygıll, onurlu tam üyeliktir. CHP bunun dışındaki hiçbir seçeneği kabul etmez." (CHP 2008)

8. "Bazı AB ülkelerinin coğrafi veya kültürel farklılıklar gibi nedenlerle Türkiye'yi tam üyelikten dışlamayı ve Türkiye'ye özel bir statü vermeyi öngören politikalarının AB'nin resmi görüşü haline dönüştürülmesi halinde, başta Gümrük Birliği olmak üzere, mevcut taahhütlerimiz gözden geçirilerek, ülkemizin çıkarlarının gerektirdiği adımlar kararlılıkla atılacaktır." (CHP 2008)

9. "Kıbrıs sorununa Rum tarafını tatmin etme amacıyla yapılacak baskılarla ve dayatmalarla çare aranması yaklaşımını reddeder. Kıbrıs Rum yönetimini Kıbrıs'ı temsil eden tek meşru devlet sayan yaklaşıma kesinlikle karşıdır. Kıbrıs Rum yönetiminin uluslararası kuruluşlarda, bu arada Avrupa Birliği’nde bütün Kıbrıs'ı temsil ettiği anlayışını kabul etmez.” (CHP 2008)

\section{RÉSUMÉS}

In this study, the Euroscepticism of the two Turkish political parties, CHP and MHP, are analysed and compared on the basis of party documents and speeches by party leaders from 2004 to 2007. The article addresses not only the types of Euroscepticism and the similarities and differences of the phenomenon, but also the causes of the different types observed by the political parties. Methodologically, the content analysis of Mayring (2008) and the difference method of John Stuart Mill (1874) are applied. The article centres from the analytical-theoretical point of view on the EU and on the party research. 
INDEX

Mots-clés : Eurosceptissisme, européanisation, partis politiques, Turquie, intégration européenne

Keywords : Euroscepticism, Europeanization, Political Parties, Turkey, European Integration Schlüsselwörter : Euroskeptizismus, Europäisierung, Politische Parteien, Türkei, Europäische Integration 